\title{
Genome-wide identification of PME genes, evolution and expression analyses in soybean (Glycine max L.)
}

Liang Wang, Yingqi Gao, Songming Wang, Qiqi Zhang and Shouping Yang*

\begin{abstract}
Background: Pectin methylesterase (PME) is one of pectin-modifying enzyme that affects the pectin homeostasis in cell wall and regulates plant growth and diverse biological processes. The PME genes have been well explored and characterized in different plants. Nevertheless, systematic research on the soybean (Glycine max L.) PME genes remain lacking.

Results: We identified 127 Glycine max PME genes (GMPME) from the soybean Wm82.a2.v1 genome, which unevenly distributed on 20 soybean chromosomes. Phylogenetic analysis classified the GmPME genes into four clades (Group I, Group II, Group III and Group IV). GmPME gene members in the same clades displayed similar gene structures and motif patterns. The gene family expansion analysis demonstrated that segmental duplication was the major driving force to acquire novel GMPME genes compared to the tandem duplication events. Further synteny and evolution analyses showed that the GMPME gene family experienced strong purifying selective pressures during evolution. The cis-element analyses together with the expression patterns of the GMPME genes in various tissues suggested that the GMPME genes broadly participate in distinct biological processes and regulate soybean developments. Importantly, based on the transcriptome data and quantitative RT-PCR validations, we examined the potential roles of the GmPME genes in regulating soybean flower bud development and seed germination.
\end{abstract}

Conclusion: In conclusion, we provided a comprehensive characterization of the PME genes in soybean, and our work laid a foundation for the functional study of GMPME genes in the future.

Keywords: Soybean, PME, Genome-wide identification, Evolution, Expression analyses, Flower bud development, Seed germination

\section{Background}

The dynamics of cell walls such as constructions, differentiations, maturations and degradations are crucial for regulating plant growth $[1,2]$. Pectin is one of the most abundant components of plant cell walls, which

*Correspondence: spyung@126.com

Soybean Research Institute, National Center for Soybean, Key

Improvement Laboratory of Biology and Genetic Improvement of Soybean (General, Ministry of Agriculture), State Key Laboratory of Crop Genetics and Germplasm Enhancement, Jiangsu Collaborative Innovation Center for Modern Crop Production, College of Agriculture, Nanjing Agricultural University, Nanjing 210095, China is associated with the porosity and hydration of the cell wall as well as the plant cell intercellular adhesion $[3,4]$. Homeostasis of pectin is balanced by different pectin-regulating enzymes $[5,6]$. Pectin methylesterase (PME, EC 3.1.1.11), so-called pectinesterase, is a hydrolytic enzyme that catalyzes the de-esterification of methyl esterified galacturonic acid residues of pectin to generate carboxyl groups and release methanol and hydrogen ions in the cell wall $[7,8]$. A high level of PME often induces the loosening of the cell wall, whereas, low PME activity tends to form a rigid cell wall [2]. Early studies turned out that the PME activity was tightly linked to distinct plant 
developmental courses, including pollen tube elongation $[9,10]$, seed germination $[11,12]$, hypocotyl growth $[13$, $14]$, fruit ripening and softening $[2,15,16]$.

In recent years, whole-genome sequencing technologies promoted the exploration of multiple gene families in diverse species [17]. Genome-wide identifications and characterizations of $P M E$ genes have been carried out in diverse plant species, including Arabidopsis [18], rice [19], maize [20], cotton [21], tomato [16] and strawberry [2]. For the gene structures, all the $P M E$ gene members contain the PME domains, and some of them meanwhile harbor the PME inhibitor (PMEI) domains [21]. For the gene functions, different $P M E$ genes play multiple roles in plants. In Arabidopsis, AtPME3 was reported to affect the metabolism of seed storage proteins during the post-germinative growth of seedlings [22]. AtPME17 was another functional pectin methylesterase gene, which was regulated by its PRO region that triggered PME activity in the resistance to Botrytis cinerea [23]. In another study, AtPME34 was turned out to contribute to heat tolerance by promoting stomatal movement in Arabidopsis [5]. Besides, Brassica campestris Male Fertility 23a (BcMF23a) was an essential pectin methylesterase gene that proved to be required for microspore development and pollen tube growth in Brassica campestris [24]. And BcPME37c is another published pectin methylesterase gene that was involved in the pollen intine formation in Brassica campestris [25].

Soybean (Glycine max L.) is an important economic crop rich in high-quality plant protein and oil [26]. The latest research carried out identification and expression analyses of the PMEI genes throughout the soybean genome [27]. However, the systematic investigation of the Glycine max PME gene (GMPME) family in soybean remains blank. In this study, we comprehensively identified 127 GmPME genes in the soybean genome. According to the classification of the PME gene members in Arabidopsis, we phylogenetically clustered and divided the GmPME members into four clades (Group I, Group II, Group III, and Group IV). Then we explored the gene structures, motif patterns, chromosomal locations and gene duplication events of the identified GmPME members. Moreover, we conducted the evolutionary analyses on the PME members among soybean and representative dicotyledons as well as monocotyledons. To survey the potential roles of GmPME genes in regulating various biological processes, we analyzed the cis-elements in the promoter regions of the GmPME genes and gene expression profiles in different tissues. High-yielding is one of the most important targets in soybean breeding [28], utilization of heterosis with the cytoplasmic male sterility (CMS) system is an effective way to fulfill this goal [29]. At present, stable soybean CMS lines, such as NJCMS1A, NJCMS2A and NJCMS5A, have been well developed and applied for soybean hybrid seed production [30]. Hence, we conducted GmPME gene expression analyses between stable soybean cytoplasmic male sterile line NJCMS1A and its maintainer NJCMS1B. Importantly, a good seed germination ability is also essential for soybean production [31]. In the current research, we investigated the expression patterns of the identified $G m P M E$ genes during seed germination processes. Besides, 17 representative GmPME genes were selected and manipulated further quantitative RT-PCR analyses during soybean flower bud development and seed germination. Overall, the current study provided a systematic characterization of the GmPME members. To a certain degree, our work facilitated future relevant gene functional research and may play a fulfilling role in promoting soybean high-yielding breeding.

\section{Results}

\section{Identification of soybean PME genes}

From the soybean Wm82.a2.v1 genome on Phytozome (https://phytozome-next.jgi.doe.gov/), we identified 127 GmPME genes originated from 20 different soybean chromosomes. Based on the chromosome orders and gene chromosomal locations, the 127 GmPME genes were designated as GmPME1 to GmPME127, respectively. (Additional file 1: Table S1).

The fundamental information of GmPME members was displayed in Table S1 (Additional file 1), including the opening reading frame (ORF) length, the protein size (aa, amino acid), the isoelectric point (pI), the molecular weight (MW), the predicted subcellular localization and the conserved domain. As is illustrated in Table S1 (Additional file 1), the protein sizes of GmPME members were ranged from 107 aa (GMPME92) to 1186 aa (GmPME54). Accordingly, the MW of GmPME members spanned from $11,780.63 \mathrm{Da}$ to $129,972.9 \mathrm{Da}$, and ORF length varied from $321 \mathrm{bp}$ to $3558 \mathrm{bp}$. Besides, the $\mathrm{pI}$ of the GmPME members ranged from 4.61 (GmPME92) to 10.12 (GmPME82). For the subcellular locations, 68 GmPME members were predicted to be located in the extracellular region, 38 in the plasma membrane, 15 were in the cytoplasmic region, three were in the nuclear region, two were in the mitochondrial region and one was in the chloroplast. And the gene coding sequences and protein sequences of the GmPME members were listed in Table S2 (Additional file 2).

\section{Phylogenetic classifications of GmPME members}

Referring to the classification of Arabidopsis thaliana PME (AtPME) members [18], we utilized the 66 published AtPME proteins (Additional file 3: Table S3) and the 127 identified GmPME proteins to construct a phylogenetic tree. Correspondingly, the GmPME gene members were divided into four clades: Group I, 
Group II, Group III and Group IV (Fig. 1). As is shown in Fig. 1 and Table S1 (Additional file 1), there were $60 \mathrm{GmPME}$ members in the Group I clade, 28 in the Group II clade, four in the Group III clade, and 35 in the Group IV, respectively.

\section{Gene structures and motif patterns of GmPME gene members}

The gene structures and motif patterns of GmPME gene members were phylogenetically clustered and depicted in Fig. 2. All the identified GmPME genes contained the PME domains and different GmPME gene members in the same clade tended to display similar gene structures. Interestingly, most GmPME gene members in the Group I, Group II and Group III clades simultaneously contained the PMEI domains (except for GMPME27, GmPME35, GmPME95 and GmPME106 in the Group I clade, GmPME96 in the Group II clade, GmPME39 and GmPME44 in the Group III clade) compared to those in the Group IV clade (Fig. 2 and Additional file 1:

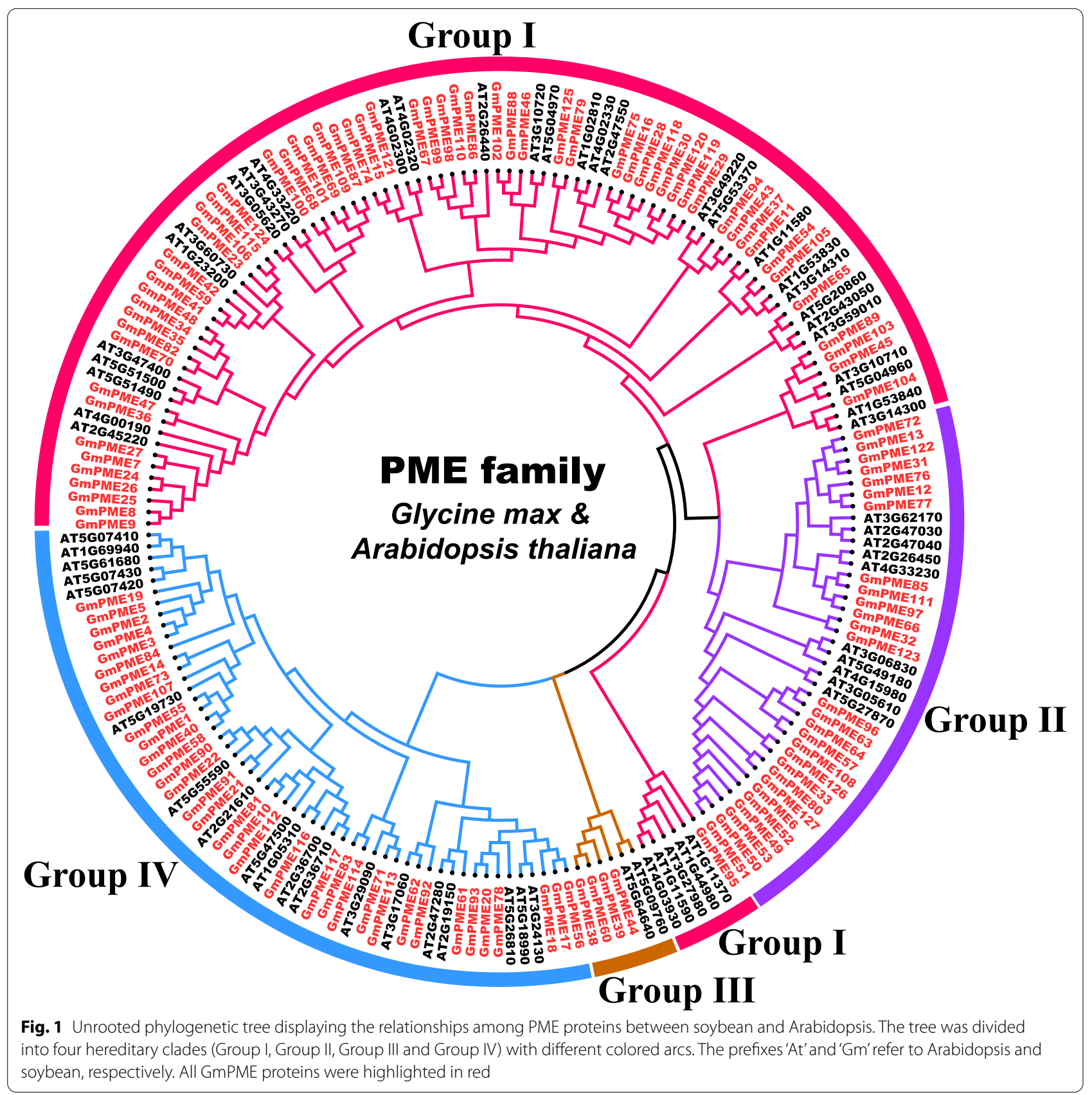




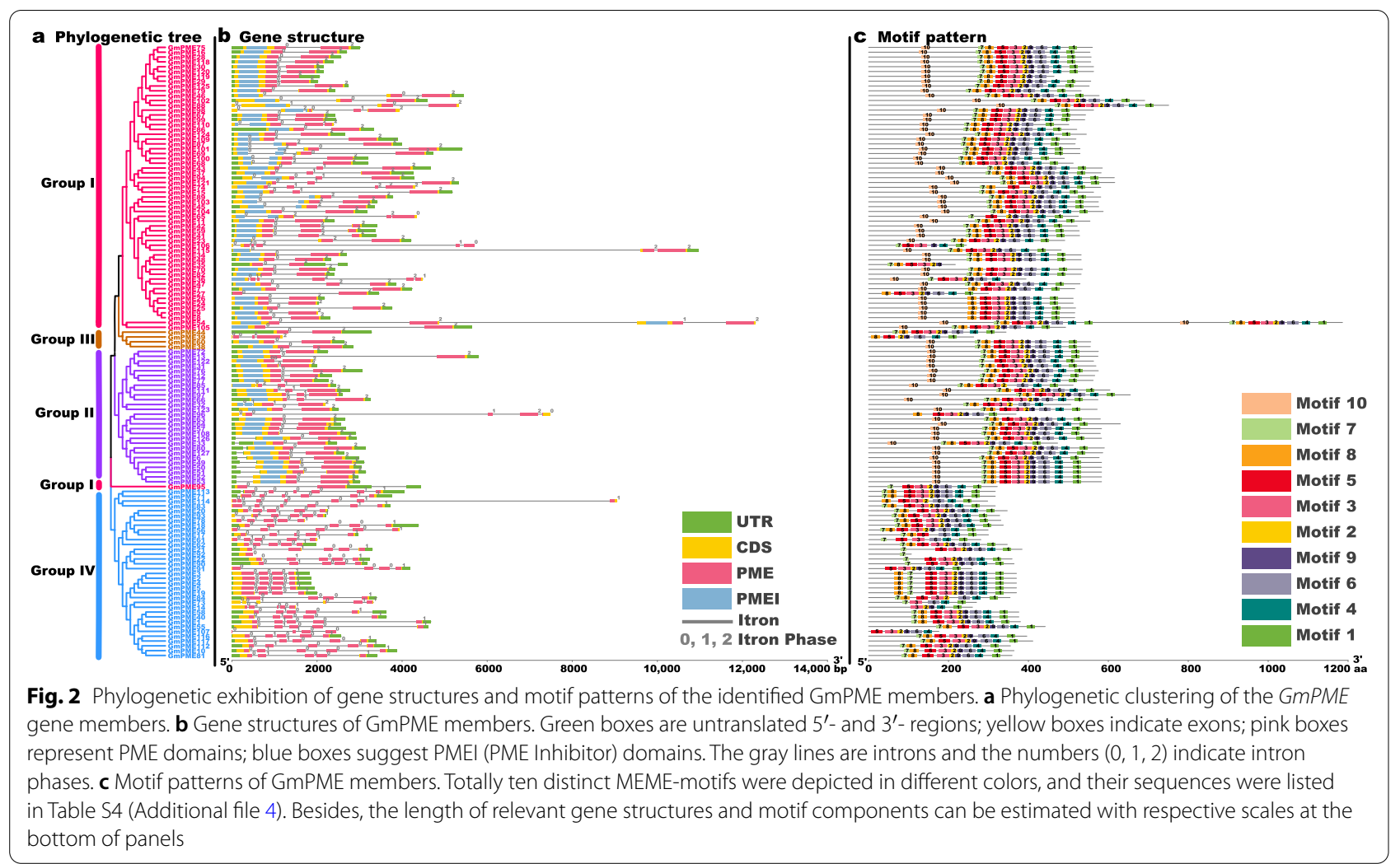

Table S1). For the exon-intron patterns, there were one to seven exons contained in GmPME gene members (one with one exon, 54 with two exons, 23 with three exons, 18 with four exons, 23 with five exons, seven with six exons and one with seven exons), and introns widely existed (Fig. 2b).

Meanwhile, the MEME-motif scanning was also carried out to better illustrate the conserved domains of the GmPME members (Fig. 2c). As is shown in Fig. 2c, ten different MEME-motifs (Motif 1 to Motif 10) were acquired. The information of the ten MEME-motifs was listed in Table S4 (Additional file 4) and Seq-Logos of the MEME-motifs were depicted in Fig. 3. Notably, Motif 10 only showed up in those GmPME members that owned the PMEI domains. Hence, we summarized that Motif 10 was corresponding to the PMEI domain, and Motif 1 to Motif 9 were linked with the PME domain in the current study (Additional file 4: Table S4). In general, the MEME-motifs in the specific clades of the GmPME gene family showed regular orders and patterns.

\section{Distributions of GMPME genes on soybean chromosomes}

The identified GmPME genes were physically anchored to the 20 soybean chromosomes (Chr 01 to Chr 20) in Fig. 4.
Overall, the GmPME genes are unevenly distributed on the chromosomes throughout the soybean genome. For example, Chr 19 contained the most GmPME genes (13 genes), whereas merely a single gene located on Chr 11, Chr 12, Chr 18 and Chr 20, respectively. Moreover, the amount of GmPME genes on soybean chromosomes did not exhibit correlations to the length of chromosomes. To better reveal the distributing tendency of GmPME genes, a series of gradient colors were endowed on soybean chromosomes. And the gradient colors were depicted based on the gene densities on soybean chromosomes, which were deduced from the gene numbers in the 300-kb genetic intervals on different soybean chromosomes (Additional file 5: Table S5). Interestingly, most identified GmPME genes tended to gather in regions with high gene densities.

\section{Duplication, synteny and evolution analyses of GmPME gene members}

Gene duplications are regarded as one of the major driving forces that promote genome evolutions [32]. Soybean is a palaeopolyploid, which under went two whole genome duplication (WGD) events near 59 and 13 million years ago, integrating a highly duplicated genome that most genes displayed multiple copies [33]. In this 
Wang et al. BMC Plant Biology

(2021) 21:578

Page 5 of 20
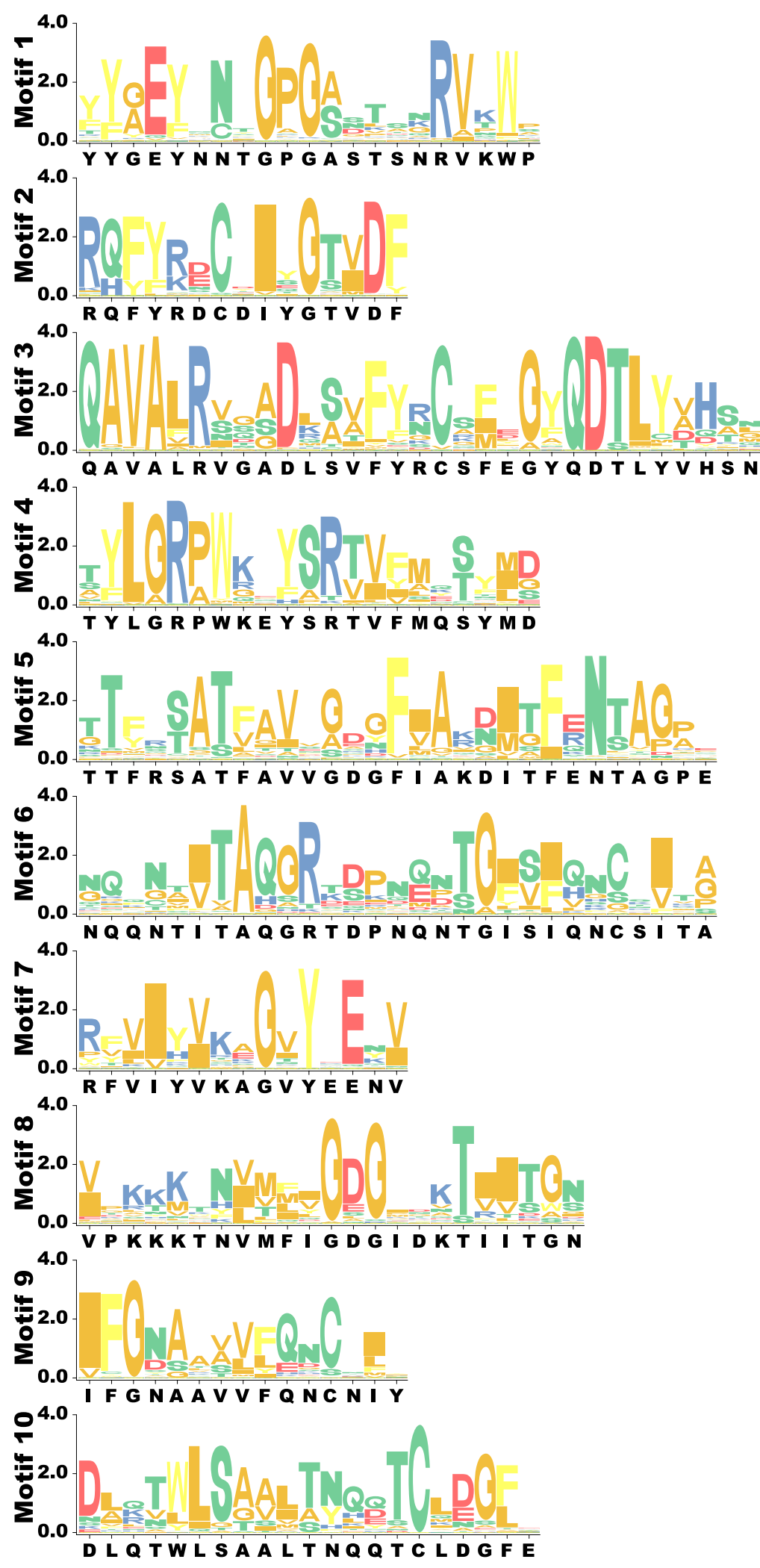

Fig. 3 Ten MEME-motif Seq Logos for the GmPME proteins 


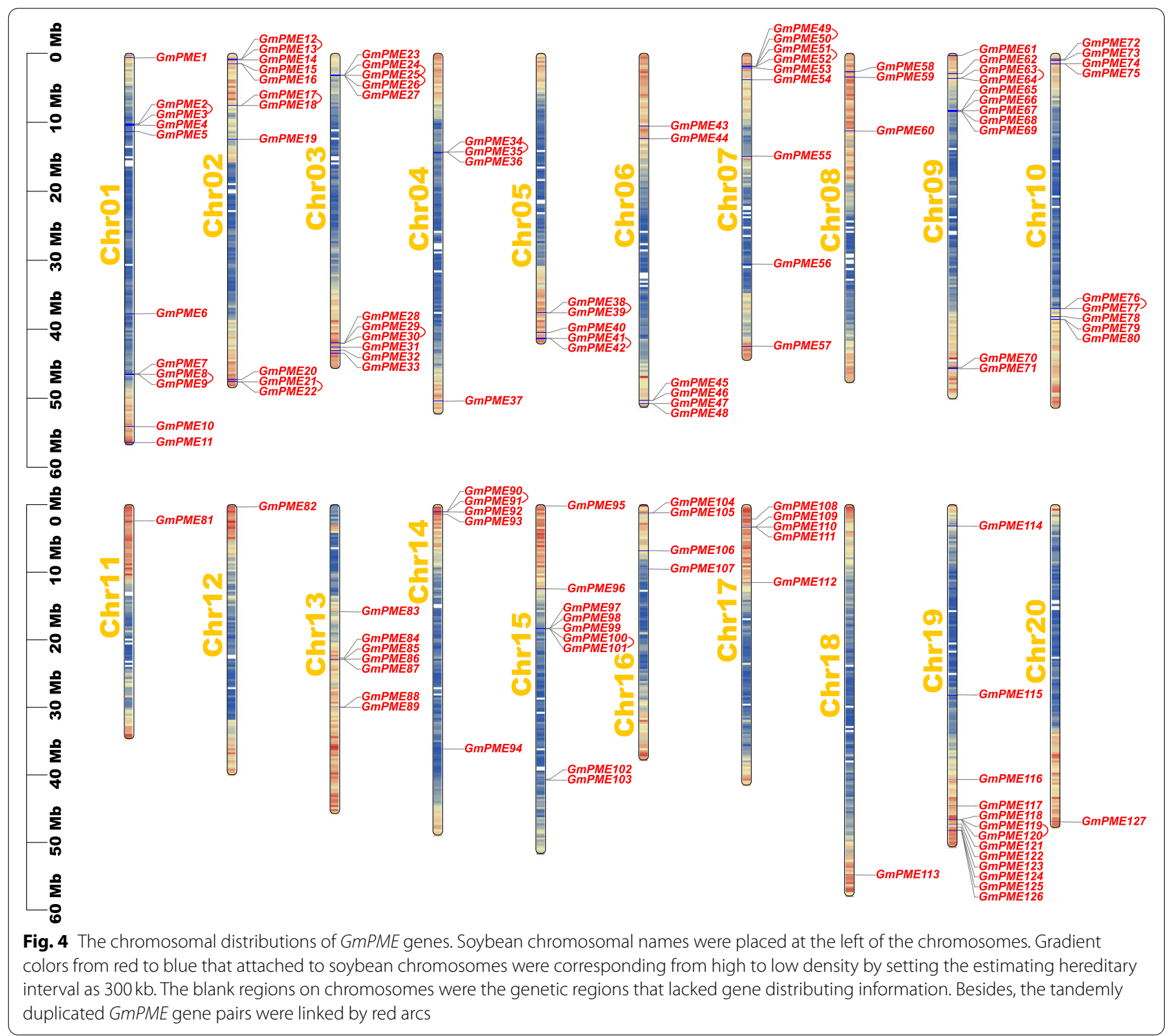

study, we calculated the synonymous substitutions (Ks) of soybean homologous gene pairs throughout the soybean genome (Additional file 6: Table S6), and the Ks frequency distribution histogram was depicted in Fig. 5a. As is shown in Fig. 5a, the distribution of Ks presented two peaks, corresponding to the recent soybean-lineage-specific palaeotetraploidization event (in gray color) and the early-legume duplication event (in black color), respectively.

Early research demonstrated that the expansions of plant gene families were mainly promoted by segmental and tandem duplication events [34, 35]. Segmental duplications often happened during polyploidization events with chromosome rearrangements, resulting in a large amount of duplicated chromosomal blocks in the genomes [36]. By contrast, tandem duplications were defined as the events that multiple adjacent homologous gene family members (two or more members) cluster on a single chromosome [37]. To gain a better overview of the two major gene duplication events in soybean, we explored and filtered the segmentally and tandemly duplicated gene pairs throughout the soybean genome (Additional file 7: Table S7 and Additional file 8: Table S8). And the duplicated gene pairs were further depicted with a series of dots in the soybean genome duplicated gene dotplot (Fig. 5b). According to the definitions of segmental and tandem duplications, the dots distributed at the diagonal line were corresponding to the tandem duplicated gene pairs, and the rest dots were the segmentally duplicated 


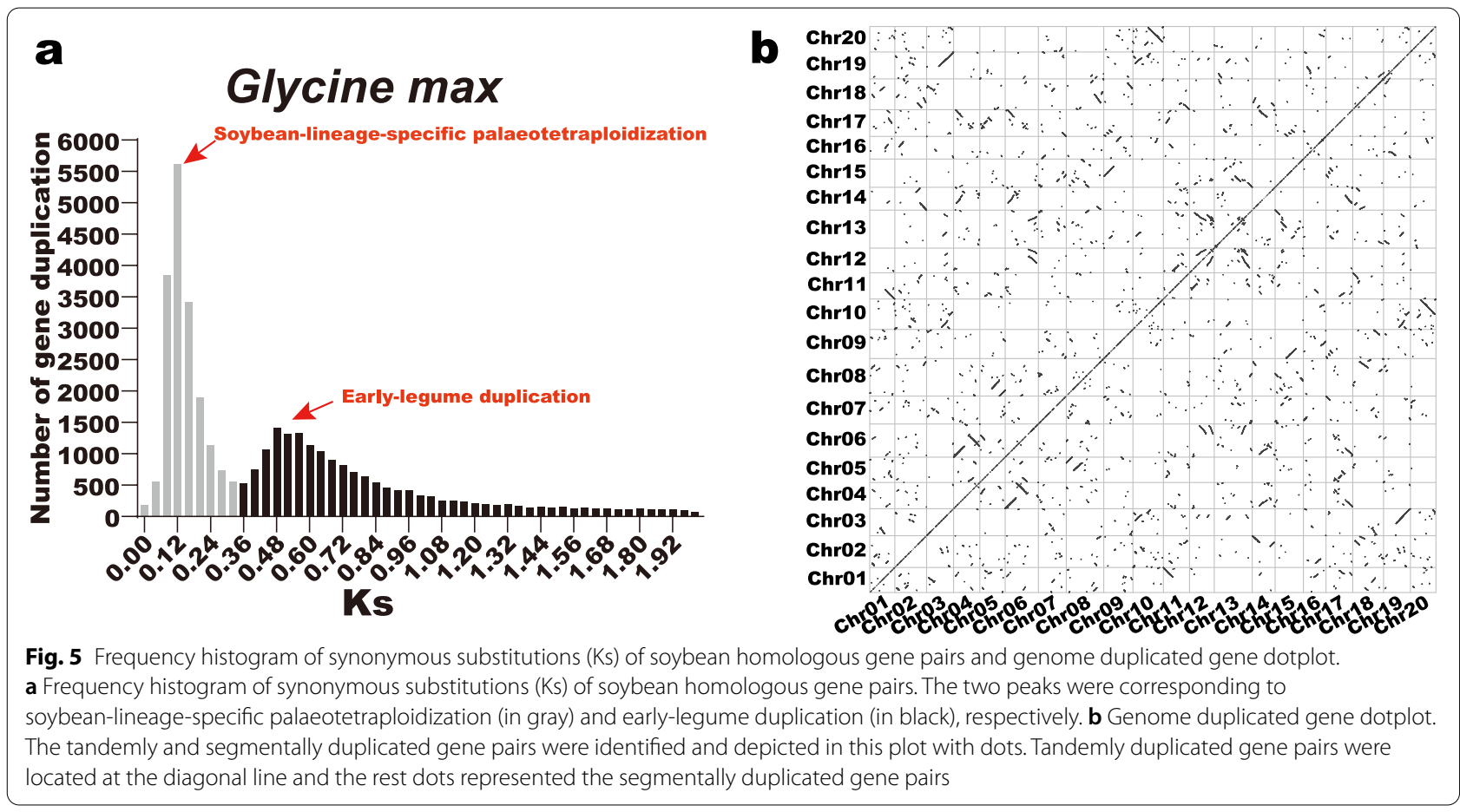

gene pairs. Our results support that soybean presents a highly duplicated genome, which is abundant in segmental and tandem duplicated gene pairs. In the GmPME gene family, we detected 18 tandemly duplicated gene pairs (Additional file 9: Table S9), which were linked by red arcs throughout 11 soybean chromosomes in Fig. 4. In contrast, we found 90 segmentally duplicated gene pairs (Additional file 9: Table S9), which were five times of tandemly duplicated ones and jointed by purple curves in collinear Circos plot in Fig. 6. Taken together, we speculated that the expansion of the GmPME gene family was both associated with tandem and segmental duplication events, whereas segmental duplications took the lead in deriving new GmPME gene members.

To seek the syntenic relations between GmPME members and the $P M E$ gene members in different species, we recruited four dicots (Arabidopsis thaliana, Glycine soja, Vigna unguiculata and Solanum lycopersicum) and two monocots (Oryza sativa and Sorghum bicolor), and carried out the synteny analyses. Consequently, 255, 149, 87, 65, 48, 38 and 33 GmPME orthologous genes were identified in Glycine soja, Vigna unguiculata, Solanum lycopersicum, Arabidopsis thaliana, Sorghum bicolor and Oryza sativa, respectively (Additional file 10: Table S10). Furthermore, we depicted six comparative syntenic graphs representing the orthologous gene pairs in soybean and the recruited species, and the $P M E$ orthologous gene pairs were linked by red curves (Fig. 7). Besides, we filtered the non-redundant GmPME genes that exhibited the syntenic relationships among soybean and the other six species (Additional file 11: Table S11), and an interactive Venn diagram of the non-redundant GmPME genes throughout the different species was displayed in Fig. 8a. Totally 12 GmPME genes contained the corresponding orthologous genes in all recruited species (Fig. 8a), which were further emphasized in bold in Table S11 (Additional file 11). The conjoint orthologous gene pairs throughout distinct species may be useful for conducting relevant evolutionary studies of GmPME genes. Importantly, the evolutionary constraints that acted on GmPME genes and their orthologous genes in the six species were also assessed by calculating the $\mathrm{Ka} / \mathrm{Ks}$ (non-synonymous substitution/ synonymous substitution) ratios (Additional file 10: Table S10). And all the acquired $\mathrm{Ka} / \mathrm{Ks}$ ratio values were used to draw a box plot in Fig. 8b. It is noteworthy that most GmPME orthologous gene pairs showed $\mathrm{Ka} / \mathrm{Ks}<1$, which indicated that the GmPME gene family may experience strong purifying selective pressures during evolution [38].

\section{Cis-elements in the promoters of GmPME genes}

To explore the potential roles of GmPME gene members participating in various soybean biological processes, we extracted the promoter region sequences of GmPME genes (the 2000 bp upstream sequences from 


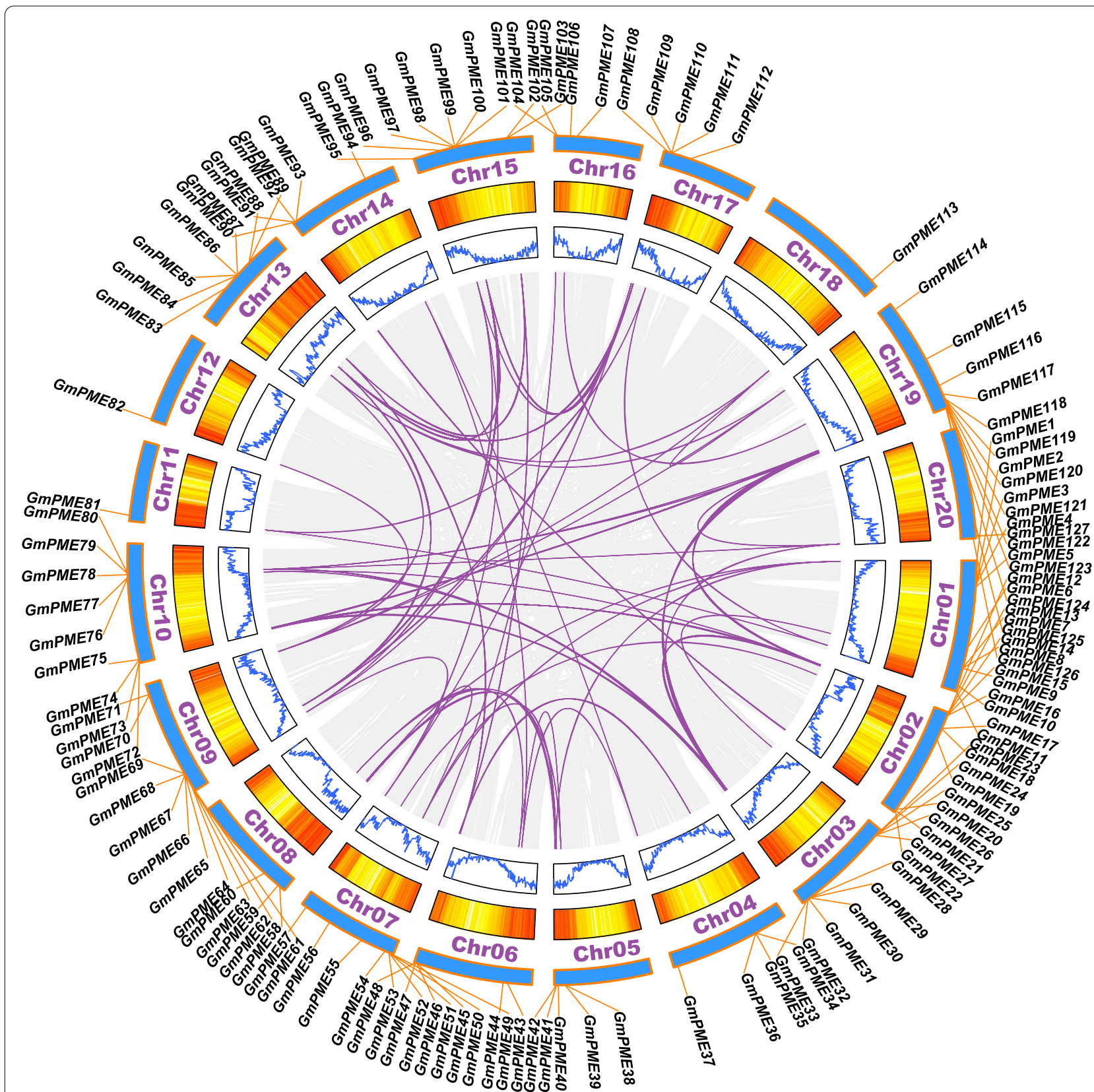

Fig. 6 Circos plot representing the collinearity of soybean homologous genes. Genome-wide collinear blocks were set as the background in gray and the duplicated GMPME gene pairs were linked and highlighted with purple curves. In addition, each soybean chromosome was attached with 300-kb gene density information depicted by heatmap and wave graph

gene initiation codons) and carried out the cis-element analyses (Additional file 12: Table S12). Furthermore, the cis-elements in gene promoter regions were proportionally illustrated (Fig. 9). As is illustrated in Fig. 9, a total of 15 distinct types of cis-elements were displayed. Importantly, cis-elements including the abscisic acid responsive element, the auxin responsive element, the salicylic acid responsive element, the MeJA responsive element, the gibberellin responsive element, the defense and stress responsive element and the low temperature responsive element were broadly existed, which suggested the GmPME gene members may link to the responses of distinct hormones and stresses. Besides, some plant growth and development associated cis-elements like the light responsive element, the anaerobic induction element, the meristem expression regulatory element, the circadian 


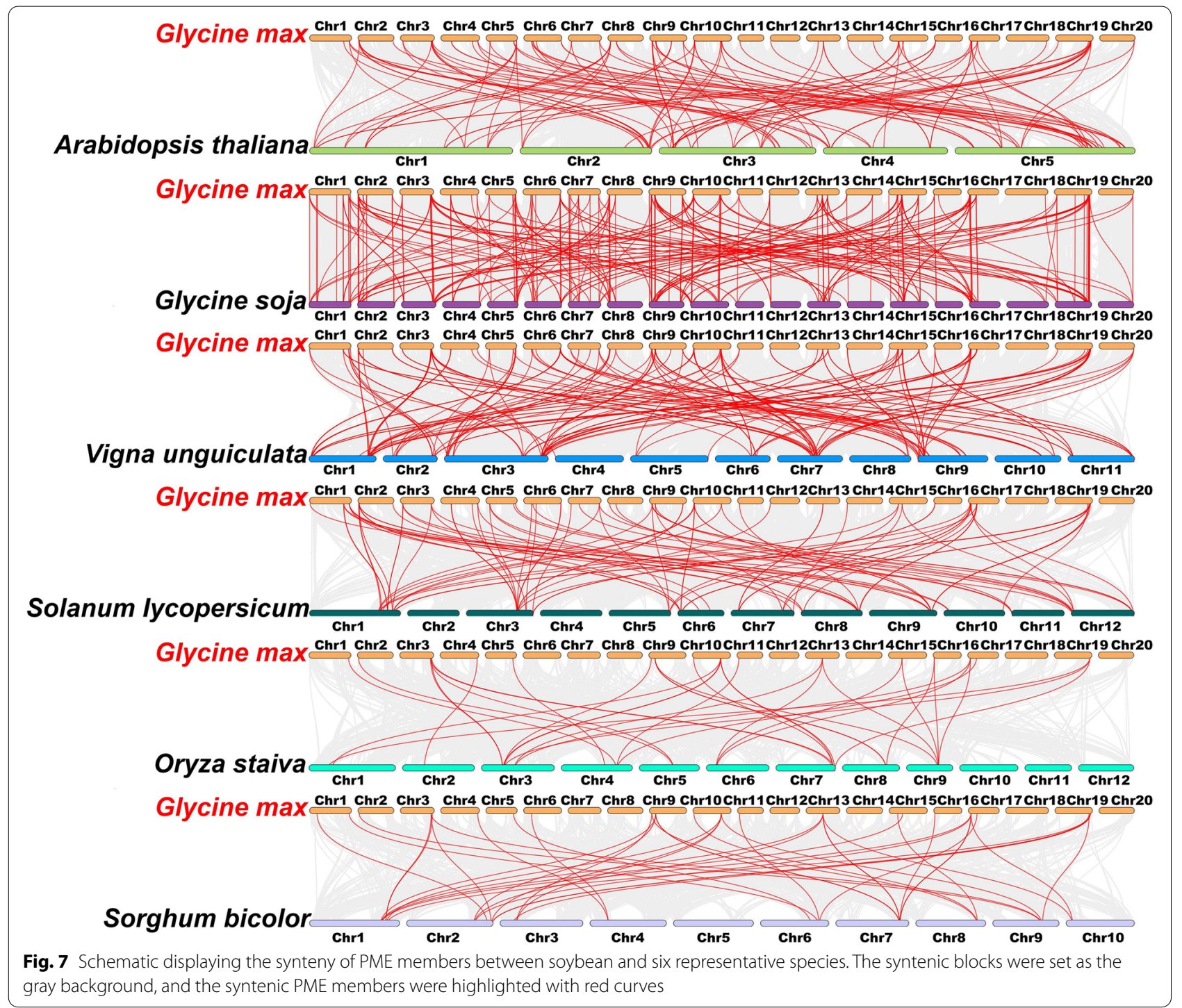

control element and the cell cycle regulation element were also detected. To sum up, the GmPME genes may take crucial roles in soybean growth and development as well as in various hormones and stress responses.

\section{Expression patterns of GmPME genes in distinct tissues}

The expression profiles of the 127 identified GmPME genes in various tissues, including shoot apical meristem (SAM), leaves, flowers, stem, root, root hairs, nodules, pods and seeds were extracted from Phytozome in Table S13 (Additional file 13). Then the extracted data were $\log _{2}$ normalized to generate a heatmap in Fig. 10a. In general, the GmPME genes in different hereditary groups or clades showed distinct expression patterns. For instance, most GmPME genes in the Group II clade displayed superior expression levels in flowers compared to other tissues. In comparison, some GmPME gene members in the Group I clade exhibited high expression levels in other tissues like root, nodules, pods and seeds. And the most GmPME genes in the Group III and Group IV clades were at relatively low expression levels throughout tissues.

To further explore the expression patterns of the GmPME genes in various tissues, we calculated the gene expression correlation coefficients among the identified GmPME gene members in Table S14 (Additional file 14). Based on the acquired correlation coefficients, a phylogenetically clustered heatmap was drawn in Fig. 10b. According to the different clades, the heatmap was separated into different blocks, and diverse groups were enclosed by solid boxes and labeled with their names in bold. As is shown in Fig. 10b, most GmPME gene members in the Group II and Group III clades showed positive 


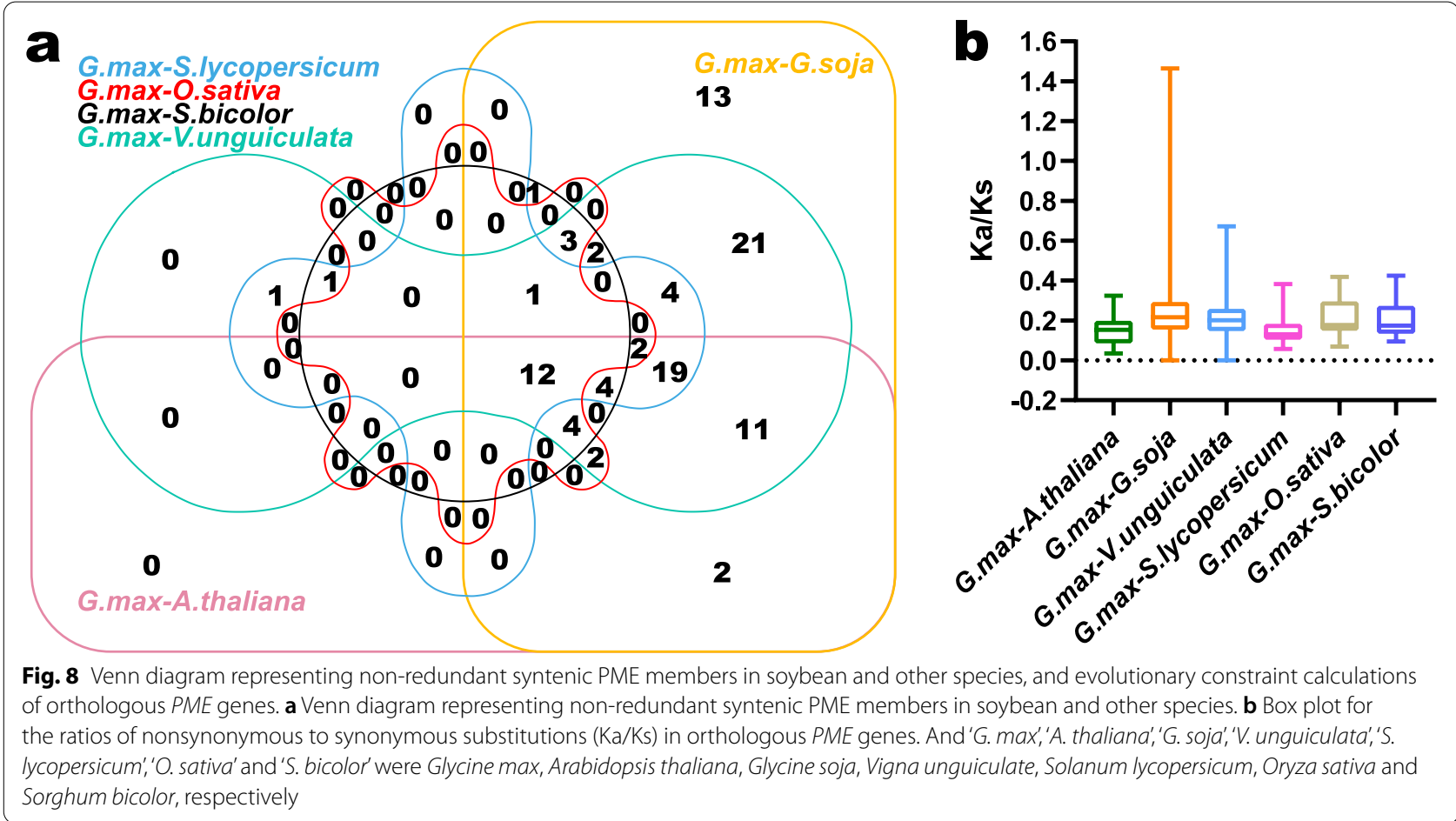

correlations with the internal members and exhibited similar expression patterns throughout the clades. By contrast, in the Group I and Group IV clades, most GmPME gene members displayed divergent expression patterns throughout the internal or external clades.

To better visualize the diverse expression patterns of GmPME genes in tissues, we concomitantly selected ten representative GmPME genes and created fancy heatmaps representing relevant gene expressions (Fig. 11). As is shown in Fig. 11a, four GmPME genes were from the Group I clade. GmPME7 mainly expressed in flowers, root and nodules; GmPME94 showed relatively high expressions in leaves, stem and pods; GmPME105 highly expressed in stem, root, root hairs, nodules and seeds; GMPME121 displayed superior expressions in SAM, stem, root, root hairs and pods. Besides, GmPME111 and GmPME122 were from the Group II clade and uniformly exhibited high expressions in flowers (Fig. 11b). GmPME38 and GmPME60 from the Group III clade were depicted in Fig. 11c. GmPME38 presented relatively high expression levels in stem, root hairs, nodules, pods and seeds. Whereas, GmPME60 displayed considerable expressions in SAM, stem, nodules, pods and seeds. The two extracted genes (GmPME71 and GmPME113) from the Group IV clade exhibited superior expressions in pods and seeds, and GmPME71 simultaneously owned relatively high expression in SAM (Fig. 11d). Moreover, the expression data of the ten extracted genes were further together normalized and integrated to acquire a heatmap to globally display the expression patterns of the representative genes in distinct clades (Fig. 11e). Overall, our findings supported that the GmPME genes diversely expressed in tissues, which may more or less highlight the important regulating roles of the key GmPME genes during soybean plant development.

\section{Expression profiling of GmPME genes in flower buds of soybean cytoplasmic male sterile line NJCMS1A and its maintainer NJCMS1B}

The utilization of crop plant heterosis is a potential way to fulfill yield breakthroughs in the future [39]. In this study, we extracted the expression profiles of the identified GmPME genes in the flower buds of soybean cytoplasmic male sterile line NJCMS1A and its maintainer NJCMS1B from the published transcriptome data (Additional file 15: Table S15) [40]. Moreover, the gene expression data was $\log _{2}$ normalized and acquired a phylogenetically clustered heatmap (Fig. 12a). As is shown in the figure, the expressions of the GmPME genes in the maintainer NJCMS1B are universally higher than those in cytoplasmic male sterile line NJCMS1A. In the Group I and Group II clades, some GmPME genes, for examples, GmPME46 and GmPME121 in the Group I clade as well as GmPME12 and GMPME122 in the Group II clade, were at relatively high expression levels both in NJCMS1A and NJCMS1B. Notably, the most highly 


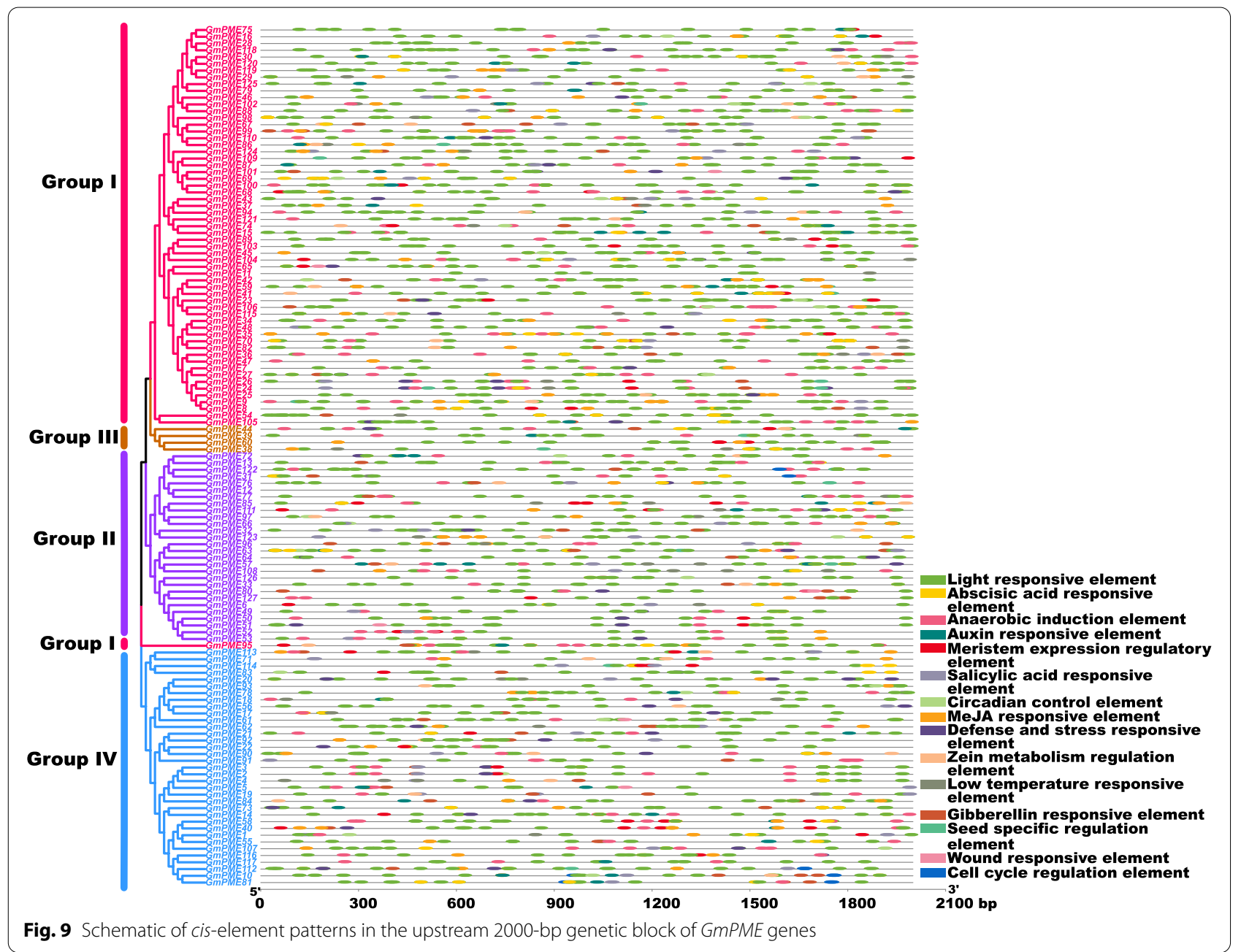

expressed GmPME genes were in NJCMS1B and gathered in the Group II clade. Whereas, in the Group III and Group IV clades, the GmPME genes were universally low expressed.

Concomitantly, the gene expression data was rowscaled with the zero-to-one method to further exhibit the expression differences of GmPME genes in cytoplasmic male sterile line NJCMS1A and its maintainer NJCMS1B (Additional file 16: Fig. S1). Importantly, in the Group I and Group II clades, we respectively selected two and six representative GmPME genes, which were at high expression levels and differently expressed in NJCMS1A and NJCMS1B (Additional file 15: Table S15), and carried out the quantitative RT-PCR analyses (Fig. 12b). As a result, all the selected genes showed significantly distinct transcript levels in NJCMS1A and NJCMS1B (* $P<0.05$ or ${ }^{* *} P<0.01$, Student's $t$-test). For instance, GmPME46 and GmPME121 in the Group I clade showed superior expressions in NJCMS1A than those in NJCMS1B (* $P<0.05)$. By contrast, the six representative genes in the
Group II clade exhibited significantly higher transcription levels in NJCMS1B than those in NJCMS1A (** $P<0.01)$. Collectively, the diverse expressions of the identified GmPME genes, especially for the representative ones, may directly or indirectly manifest their important roles in regulating flower bud development in soybean cytoplasmic male sterile line NJCMS1A and its maintainer NJCMS1B.

\section{Expression patterns of GmPME genes during soybean seed germination}

Previous studies demonstrated that the PME activity may contribute to the temporal regulations of mechanical properties of the cell walls, and thereby affect seed germination $[11,41]$. In the current investigation, the expression data of the identified GmPME genes in the embryonic axes during soybean seed germination was derived from the transcriptome data (Additional file 17: Table S16) [42]. As a result, the expression profiles of $121 \mathrm{GmPME}$ genes were acquired, and the extracted data were $\log _{2}$ 


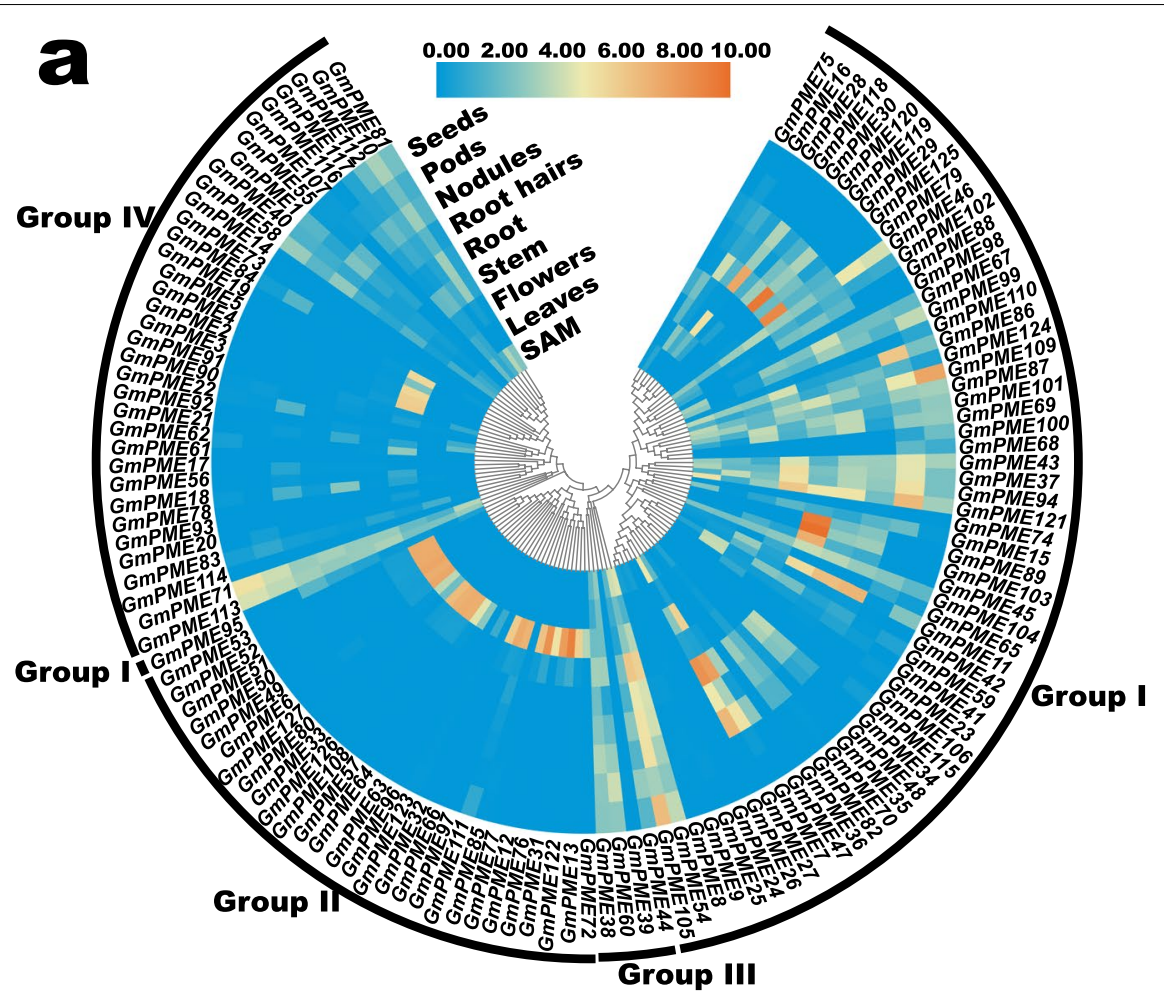

b

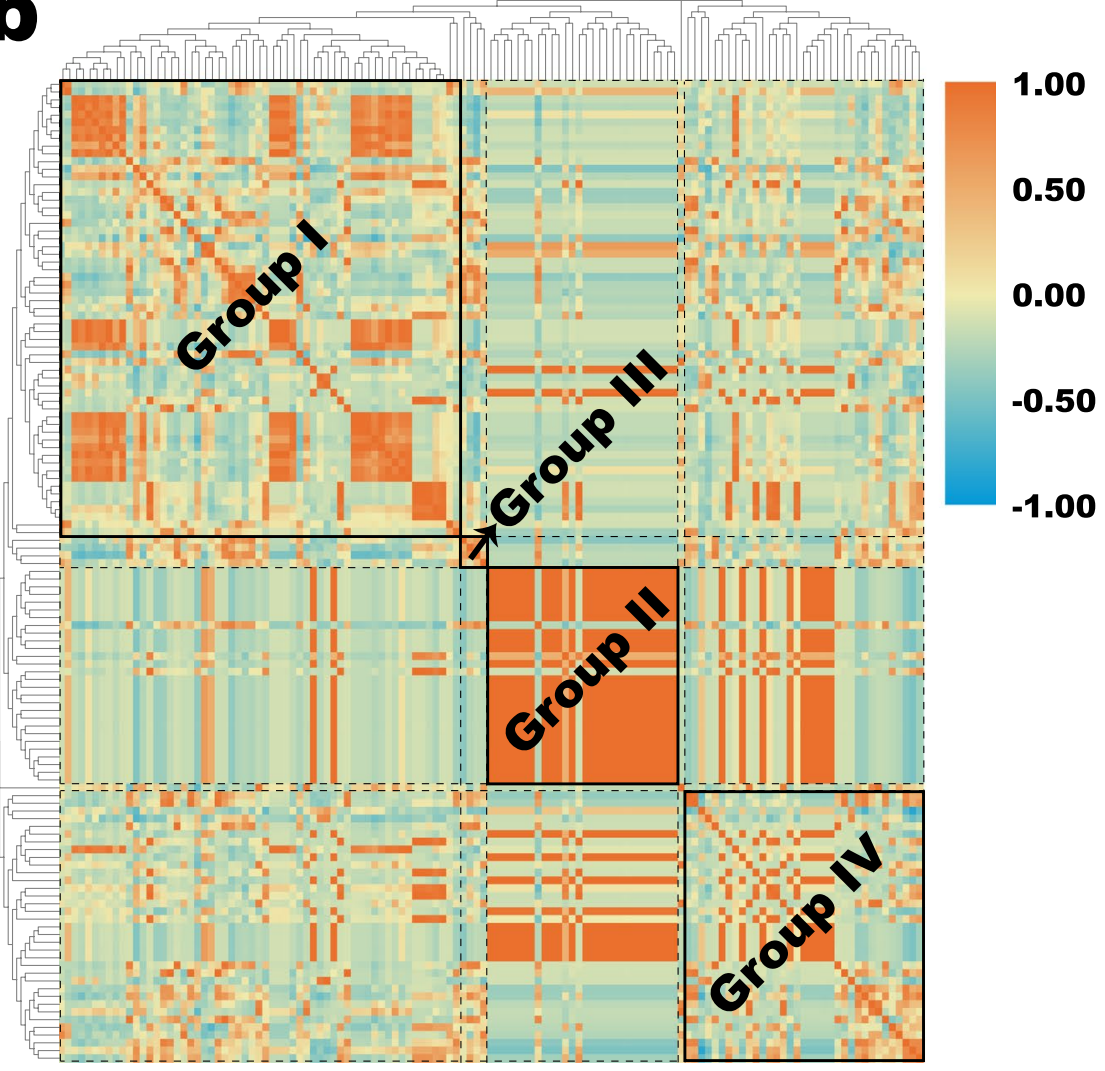

Fig. 10 Expression profiling of GMPME genes in multiple tissues on Phytozome. a Expression profiles of GmPME genes in multiple tissues on Phytozome. The extracted FPKM values were $\log _{2}$ normalized to display the heatmap. SAM: Shoot apical meristem. $\mathbf{b}$ Gene expression correlation heatmap of the GMPME gene in diverse tissues. Red: positively correlated; blue: negatively correlated 


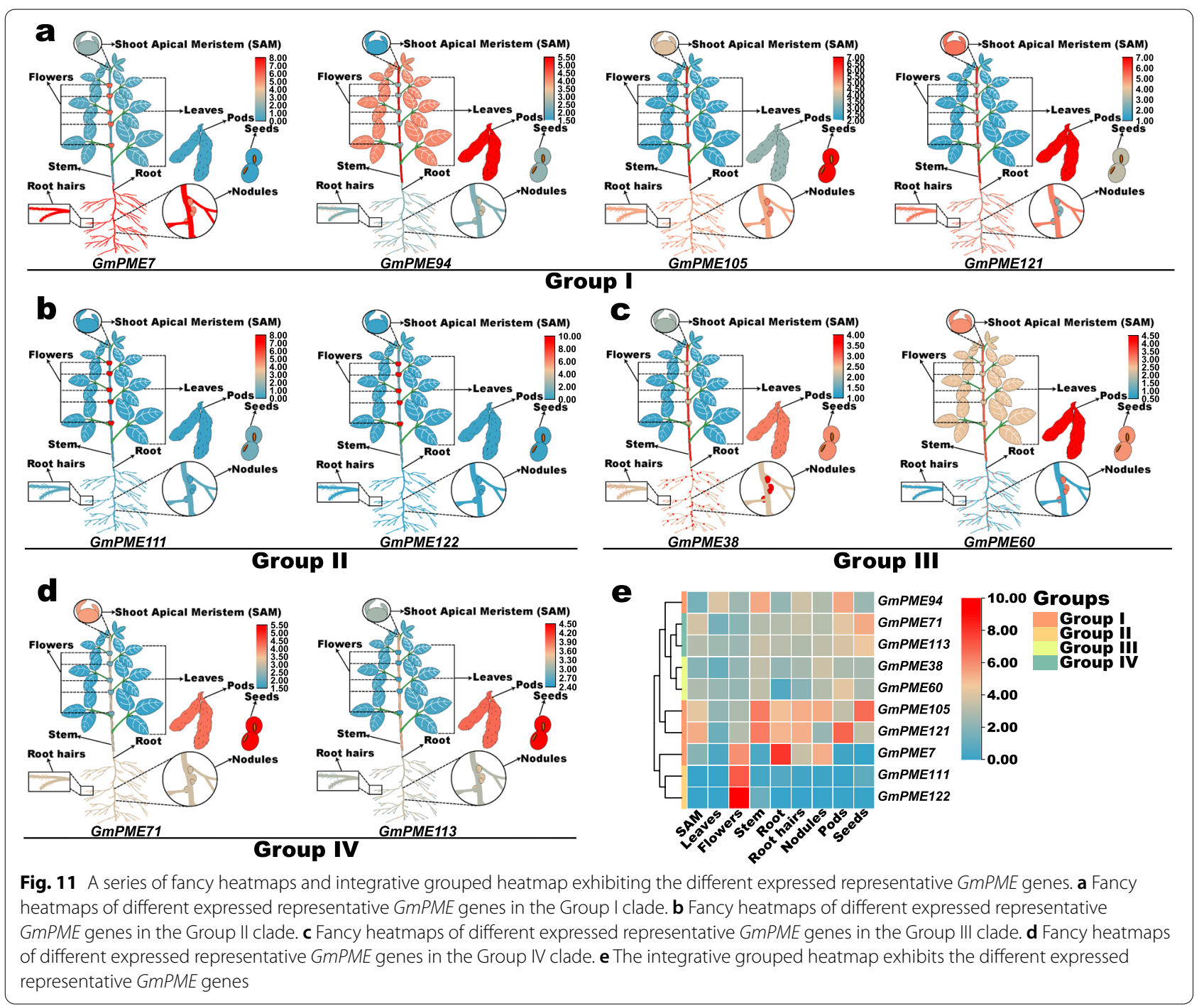

normalized to draw a phylogenetically clustered heatmap (Fig. 13a). And five time points (dry, 3 HAI (hours after imbibition), $6 \mathrm{HAI}, 12 \mathrm{HAI}$ and $24 \mathrm{HAI}$ ) were investigated during soybean seed germination. Overall, most GmPME genes showed relatively low expression levels during soybean germination. Whereas, some GmPME genes were dramatically up-regulated over time throughout different clades, which may indicate these genes participate in the regulations of soybean seed germination.

Likewise, the gene expression heatmap was additionally row-scaled with the zero-to-one method to show the expression variations of each GmPME gene during soybean seed germination processes (Additional file 18: Fig. S2). To further confirm whether the GmPME genes regulate the soybean seed germination processes, ten representative GmPME genes, whose expression levels were relatively high at diverse time points, were carefully selected from different genetic clades (Group I, Group II and Group IV) and carried out the quantitative RT-PCR validations (Fig. 13b). As a whole, most selected GmPME genes (except for GmPME113) were remarkably upregulated at the very beginning during soybean seed germination (3 HAI or $6 \mathrm{HAI}$ ). Besides, the selected genes exhibited various expression patterns, which may indicate they regulate seed germination in different patterns (Fig. 13b).

\section{Discussion}

Pectin methylesterases (PMEs) contribute to the balance of solidifying and softening of plant cell walls, which are crucial for plant growth and diverse biological processes $[43,44]$. The rapid development of whole-genome sequencing technologies boosted up the recent identifications and analyses of $P M E$ gene families in different plants. Despite the soybean (Glycine max) genome having been well sequenced, however, there were few reports 

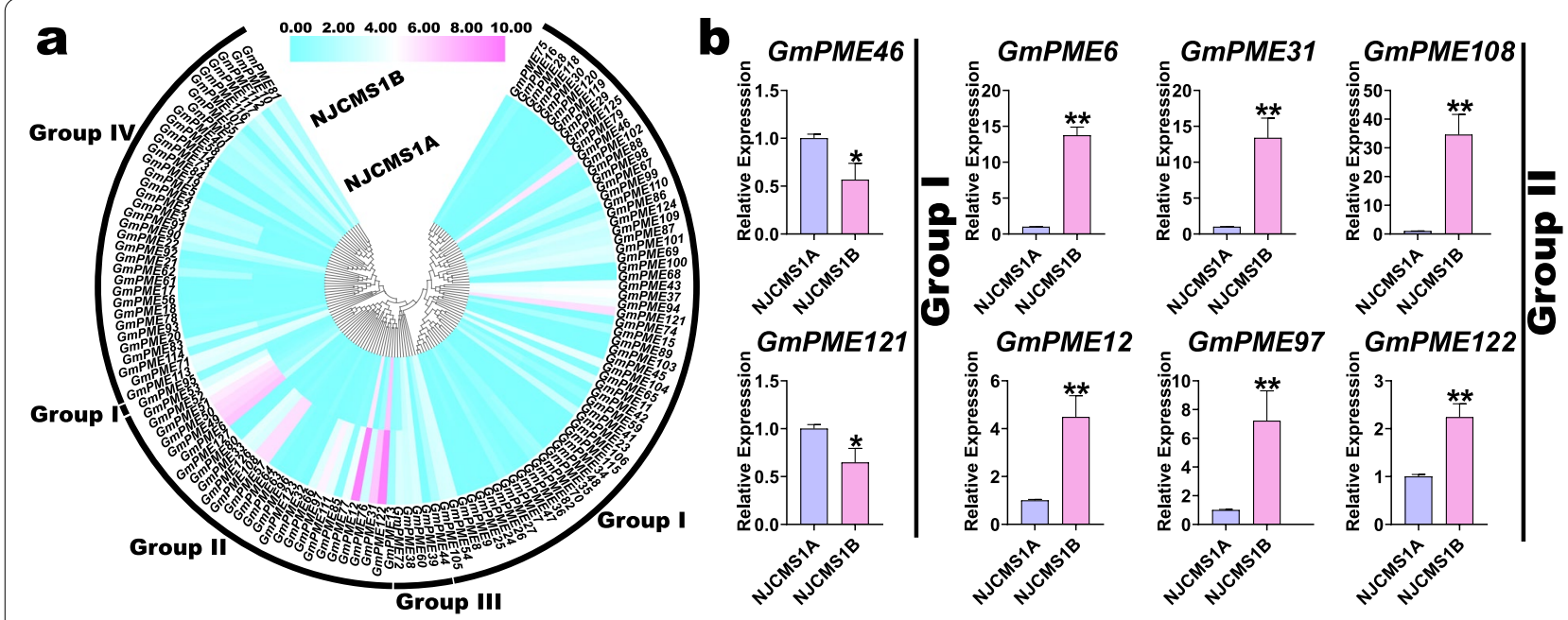

Fig. 12 Expression profiling of GMPME genes in soybean flower buds from cytoplasmic male sterile line NJCMS1A and its maintainer NJCMS1B. a Expression profiles of GMPME genes in soybean flower buds from NJCMS1A and NJCMS1B. b The quantitative RT-PCR analyses of eight selected representative GMPME genes from the Group I and Group II clades. The results of quantitative RT-PCR were normalized to the GmActin housekeeping gene. The error bars indicated the standard deviations and the values in plots were corresponding to the mean \pm standard deviation (SD) of three independent biological replicates. Asterisks demonstrated that the selected GmPME genes were significantly up- or down-regulated compared with those in soybean flower buds from NJCMS1A ( $P<0.05,{ }^{* *} P<0.01$, Student's $t$-test)
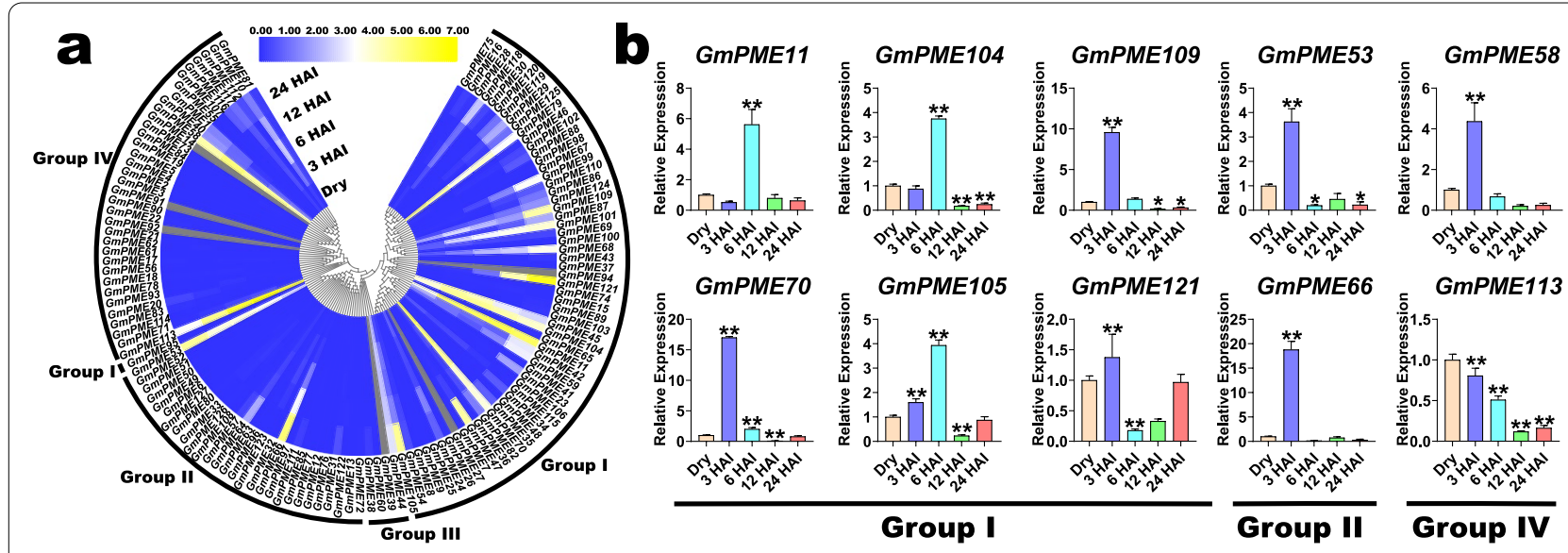

Fig. 13 Expression profiling of GMPME genes in soybean embryonic axes during seed germination. a Expression profiles of GmPME genes in soybean embryonic axes during seed germination. HAl: hours after imbibition. GMPME genes that lacked expression information in the transcriptome data were depicted with the gray color in the heatmap. b The quantitative RT-PCR analyses of ten selected representative GmPME genes from the Group I, Group II and Group IV clades. The results of quantitative RT-PCR were normalized to the GmActin housekeeping gene. The error bars indicated the standard deviations and the values in plots were corresponding to the mean \pm standard deviation (SD) of three independent biological replicates. Asterisks demonstrated that the selected GMPME genes were significantly up- or down-regulated compared with those in the dry soybean embryonic axes $\left({ }^{*} P<0.05,{ }^{*} P<0.01\right.$, Student's $t$-test)

on Glycine max PME (GmPME) genes. In the current study, we comprehensively explored and characterized the $P M E$ gene family in soybean.

Totally 127 GmPME genes were identified from the soybean Wm82.a2.v1 genome and were later divided into four hereditary clades (Fig. 1 and Additional file 1: Table S1). Soybean underwent two whole genome duplication events (Fig. 5a) and owned a highly duplicated genome coupled with multiple gene copies. The number of $P M E$ genes are varied in species. Here, we acquired more $P M E$ gene members in soybean than those reported in flax (105 members) [45], Arabidopsis (66 members) [18], rice (59 members) [19], strawberry (54 members) [2] and maize (43 members) [20]. Plant genome evolutions often along with segmental and tandem duplications that resulted in the expansions of 
different gene families [46]. As is depicted in Figs. 4 and 6 , segmental duplications mainly drove the expansion of $G m P M E$ genes, and this was also following the results in the soybean genome duplication dotplot (Fig. 5b). Interestingly, the identified GmPME genes are unevenly distributed on 20 soybean chromosomes, which might be caused by gene replications or partially fragmental gene duplications of the genome during soybean evolution $[21,47]$.

Introns were reported to be associated with the expansions of gene families during plant evolutions, which usually raised at the earlier stages of gene family expansion and gradually wiped over time [17, 48, 49]. Besides, the enlargement of introns was regarded as an effective way to gain new gene functions [50]. By investigating the gene structures, we noticed that introns broadly existed in GmPME genes (Fig. 2). For instance, GmPME54, GmPME105, GmPME106 and GmPME115 in the Group I clade; GmPME13 and GmPME96 in the Group II clade; GmPME114 in the Group IV clade contained large-spanning introns, which may indicate that they were relatively novel GmPME genes. In general, GmPME genes that clustered in the same clade exhibited analogous exon-intron patterns and conserved domain components (Fig. 2), and this may demonstrate that they conduct analogical gene functions. Notably, most GmPME gene members in the Group I, II and III clades also have PMEI conserved domains at the $\mathrm{N}$-terminuses (Fig. 2), which were similar to the $P M E$ gene members reported in cotton, maize and strawberry $[2,20,21]$. Compared to the C-terminuses, the $\mathrm{N}$-terminuses displayed relatively variable characteristics during evolution processes. GmPME gene members that contained both PME and PMEI conserved domains may cause structural as well as functional alterations of PMEs. And to some extent, the conservation of PMEI domains also facilitates keeping the fundamental functions of the gene family, improves the gene diversities, and shrinks the selection pressures during evolution [21].

To further explore the hereditary mechanisms of GmPME genes, we carried out synteny analyses of $P M E$ gene members between soybean and six representative species (four dicots and two monocots) in Fig. 7. Compared to those in monocots, $P M E$ genes in dicots presented superior syntenic relations with GMPME genes. And Glycine soja and Glycine max (soybean) exhibited the best synteny, which highlighted the evolutionary differentiation in distinct species [17]. In this study, evolutionary constraints acting on the GmPME gene family were evaluated by calculating the $\mathrm{Ka} / \mathrm{Ks}$ ratios of the orthologous $P M E$ gene pairs. Despite most ratios showing $\mathrm{Ka} / \mathrm{Ks}<1$, whereas, we also found that some orthologous gene pairs between Glycine soja and Glycine max exhibited $\mathrm{Ka} / \mathrm{Ks}>1$ (Additional file 10: Table S10), which manifested these orthologous $P M E$ gene pairs underwent positive selections [51].

The cis-element analyses in the current study further supported the potential roles of the identified GmPME genes in regulating soybean developments and responses to various biological processes (Fig. 9). By exploring the expression patterns of GmPME genes in different tissues, we acquired a diverse transcript abundance (Fig. 10) and manifested that they might dissent in gene functions. Besides, the homologous GmPME genes in the specific clade may have similar functions. Remarkably, most GmPME gene members in the Group II clade showed alike expression patterns (Fig. 10b), and they tended to show high gene expression levels in the flower buds in NJCMS1B (Fig. 12a). In Arabidopsis, $P M E$ genes in the group 2 clade were highly or uniquely expressed in the flower buds and were proved to be involved in pollen maturation and pollen tube growth [18]. In cotton, transcriptome analysis revealed that most $P M E$ genes expressed higher in flower buds of the fertile individuals than those in the CMS-D8 line [52]. Moreover, semi-qRT-PCR showed that 13 maize PME/ $P M E I$ genes were differentially expressed in the anthers of CMS fertile and sterile plants [20]. The utilization of heterosis with the CMS systems is a feasible way to increase soybean yield [29, 30,40]. In this study, we conducted gene expressing dissection between soybean cytoplasmic male sterile line NJCMS1A and its maintainer NJCMS1B by investigating previous transcriptome data (Fig. 12a and Additional file 15: Table S15) [40]. Similar to the findings in other plant species, most GmPME genes exhibited higher transcript levels in NJCMS1B than those in male sterile line NJCMS1A, and most high expression genes were gathered in the Group II clade [20,52]. Importantly, distinct representative GmPME from the Group I and II clades were selected and conducted the quantitative RT-PCR analyses. As a whole, the expression patterns of selected GmPME genes displayed by quantitative RT-PCR matched the transcriptome data, which further supported the validity of the early transcriptome analysis [40].

Seed germination is one of the principal processes in the plant life cycle [53]. Seeds with higher vigor and quality guaranteed crop yield [17]. PMEs play an important role in plant developments, including the regulations of seed germination $[2,12]$. A previous study in yellow cedar demonstrated that PME activity positively correlated with seed germination performance [54]. In Arabidopsis, PME activities rarised until the completed time of testa rupture, then gradually decreased with the weakening and rupture of endosperm [11]. Besides, the pectin-modifying enzyme genes exhibited high transcript levels during the first $24 \mathrm{~h}$ of Arabidopsis seed germination [12, 55]. In the current research, we explored the expression patterns of GmPME genes in soybean embryonic axes during seed germination 
[42]. Notably, different GmPME genes displayed diverse expression patterns throughout clades, which highlighted their distinct roles in regulating soybean seed germination (Fig. 13a). Simultaneously, we selected ten representative GmPME genes from different clades and conducted the quantitative RT-PCR analyses. In general, the expressions of most selected GmPME genes, except for GmPME113, were remarkably up-regulated at early time points of soybean germination than dramatically down-regulated over time, which highlighted the characteristics of the selected GmPME genes in temporal regulation during seed germination. It is worth noting that the transcript levels of the selected GmPME genes in the quantitative RT-PCR presented divergences compared with those expression patterns displayed in transcriptome data (Fig. 13a and Additional file 17: Table S16) [42]. For example, GmPME105, GmPME109 and GmPME121 in the Group I clade, as well as GmPME66 in the Group II, showed earlier gene upregulations compared to those in the transcriptome data (Fig. 13a), which may produce by the seed soaking step in this study that boosted relevant gene up-regulation in advance [17]. Besides, the rest selected GmPME also displayed more or less differences with the transcriptome data. The possible cause may be due to soybean varieties used in the previous study (cv. 'BRS 284') and the present research (cv. 'Williams 82') exist hereditary disparities [42]. Taken together, we speculated that relevant GmPME genes may take possible roles in modulating soybean seed germination.

In summary, we conducted a comprehensive survey on GmPME genes and the findings in the current study may provide reference points in future gene function studies. In this study, we demonstrated the potential roles of the identified GmPME genes during flower bud developments and seed germination by carrying out gene expression profiling. Nevertheless, the GmPME genes still need to be endowed with further functional investigations to illustrate their detailed roles in diverse biological courses.

\section{Conclusions}

The $P M E$ gene families have been examined in several plant species and related to various important developmental and biological processes. In comparison, associated research about the soybean $P M E$ gene family is still limited. This study provided a comprehensive identification and characterization of the GmPME genes, including gene structures and conserved domain properties, gene chromosomal location depictions, gene family member phylogeny and evolutionary investigations, ciselement exhibitions in the gene promoter regions, gene expression patterns in different tissues, gene expression profiling during flower bud development as well as seed germination processes. In conclusion, our work could pave the way for future functional study of $P M E$ genes in soybean, especially served valuable references for the regulations of $P M E$ genes during soybean flower bud development and seed germination.

\section{Methods}

\section{Identification of $P M E$ genes in soybean}

Gene identification was according to the method of Wang et al. [17]. Soybean genome (the Wm82.a2.v1 version) and its annotation file were obtained from Phytozome (https://phytozome-next.jgi.doe.gov/). All the reported AtPME protein sequences were downloaded from TAIR (https://www.arabidopsis.org/) and set as the query sequences to extract the most representative GmPME protein sequences by TBtools software [56]. Moreover, the extracted GmPME proteins were further checked by NCBI BLASTp (https://blast.ncbi.nlm.nih.gov/Blast.cgi? PROGRAM=blastp\&PAGE_TYPE=BlastSearch\&LINK LOC=blasthome). The conserved domains of GmPME proteins were analyzed by SMART (http://smart.embl. de/). Proteins less than 100 aa (amino acid), without the PME domains or with obvious errors were manually filtered. The ExPASy online tool (http://expasy.org/tools/) was adopted for examining the molecular weight (MW) and the isoelectric point (pI) of the GmPME proteins, and CELLO (http://expasy.org/tools/) were recruited for GmPME gene subcellular localization predictions.

\section{Phylogenetics and classifications of GmPME members}

The phylogenetic tree was deduced by MEGA 7.0 (https:// www.megasoftware.net/) with the neighbor-joining (NJ) method [57]. The AtPME and GmPME protein sequences were aligned by using the MUSCLE approach [58]. The phylogenetic tree was constructed with the Poisson model, pairwise deletion, and 1000 bootstrap replications. The GmPME members were divided into distinct groups based on the classifications of AtPME members in Arabidopsis. Both FigTree (http://tree.bio.ed.ac.uk/softw are/figtree/) and Adobe Illustrator (https://www.adobe. $\mathrm{com} /$ products/illustrator/free-trial-download.html) were recruited to decorate the original phylogenetic tree.

\section{Gene structure and motif pattern illustrations}

Based on the soybean genome annotation file and the acquired SMART conserved domain information, we displayed the GmPME gene structures by TBtools [56]. For motif patterns, the GmPME proteins were submitted and carried out the conserved motif scanning on the MEME website (http://meme-suite.org/tools/meme) by setting the MEME-motif as ten. And the obtained MEMEmotifs were ulteriorly matched to the conserved domains (Additional file 4: Table S4). Besides, the Seq Logos of the MEME-motifs were illustrated by TBtools. Adobe Illustrator software was adopted to polish the graphs. 
Chromosomal location, duplication, synteny and evolution analyses of GmPME gene family

According to the soybean genome annotation file, we extracted the 300-kb hereditary interval gene densities (Additional file 5: Table S5) and further transformed them into the gradient colored heatmap on each soybean chromosome. The chromosomal locations of GmPME genes were depicted with TBtools based on the soybean genome annotation file [56]. Gene family duplication analysis was carried out by calculating the synonymous substitutions (Ks) of the homologous gene pairs with TBtools. The Ks frequency distribution histogram was used to evaluate soybean whole genome duplication (WGD) events. Soybean whole genome segmentally and tandemly duplicated gene pairs were detected and further depicted in the genome duplicated genes plot by TBtools. For GmPME segmentally and tandemly duplicated homologous gene pairs, we linked them by the red arcs in the gene chromosomal location plot and the purple curves in the collinear Circos plot, respectively [59]. Besides, synteny analyses among the $P M E$ gene members of soybean and other species on Phytozome including Arabidopsis thaliana (TAIR annotation release 10), Glycine soja (V1.1), Vigna unguiculata (V1.1), Solanum lycopersicum (ITAG3.2), Oryza sativa (MSU annotation release 7.0) and Sorghum bicolor (V3.1.1) were also explored. And syntenic graphs of multiple species were generated by TBtools. Moreover, GmPME genes that contained orthologous genes in distinct species were summarized by the Venn diagram. The ratios of nonsynonymous substitution (Ka) to synonymous substitution (Ks) of GmPME orthologous gene pairs were computed with TBtools, and the acquired results were presented by box plot with Graphpad Prism 8 (https://www.graphpad. com/scientific-software/prism/). The derived graphs were further edited by Adobe Illustrator software.

\section{Cis-element analyses of GmPME gene promoter regions}

Based on the extractions of the GmPME gene upstream 2000 bp sequences with TBtools, the cis-elements in gene promoter regions were explored with PlantCARE online tools (http://bioinformatics.psb.ugent.be/webtools/plant care/html/) [60]. The schematic of cis-element distributions in GmPME gene promoter regions was displayed by TBtools. Adobe Illustrator was used to modifying the graph.

\section{Expression profiling of GmPME genes}

The expression profiles of GmPME genes in various tissues were extracted from transcriptome data on Phytozome. Additionally, the expression correlation matrix of GmPME genes in tissues was calculated by Omicshare online tools (https://www.omicshare.com/tools/Home/ Soft/getsoft). And the acquired matrix was adopted to generate the correlation heatmap by TBtools [56]. To better exhibit gene expression patterns in tissues, distinct representative GmPME genes were selected from different hereditary clades to draw a panel of gene expression fancy heatmap by using TBtools. Besides, the transcriptional levels of the identified GmPME genes in flower buds of soybean cytoplasmic male sterile line NJCMS1A and its maintainer NJCMS1B as well as the expression patterns of the GmPME genes in soybean embryonic axes during seed germination were investigated from early research data [40, 42]. All the acquired transcriptome data were assessed by the FPKM (fragments per kilobase million) values. And the FPKM values were $\log _{2}$ normalized, then using TBtools to depict associated gene expression heatmaps. Adobe Illustrator was used to modifying the graph.

\section{Plant materials and samplings}

Soybean cytoplasmic male-sterile line NJCMS1A was derived from the consecutive backcross approaches with the donor parent N8855 and the recurrent parent N2899 (further designated as NJCMS1B) cultivars [61]. And cultivar Williams 82 was adopted for seed germination assay. All plant materials were planted and harvested in 2019 at Dangtu Experimental Station, National Center for Soybean Improvement, Nanjing Agricultural University, Dangtu, Anhui, China. Samplings were originated from three independent individuals of the plant materials. During flowering growth stages, different size flower buds of NJCMS1A and NJCMS1B were collected and frozen in liquid nitrogen and then stored at $-80^{\circ} \mathrm{C}$ [29]. According to Wang et al., 40 intact soybean seeds for germination assay were selected from the independent plants and dried for 3 days at $40^{\circ} \mathrm{C}$ to keep uniform moisture [17]. And the selected seeds were pre-disinfected by $0.05 \%$ potassium permanganate solution for $5 \mathrm{~min}$ and washed with deionized water. Seed germination was performed by using the germination pouches (PhenoTrait Technology Co., Ltd.) followed by the instruction book: the disinfected seeds were soaked in deionized water for $2 \mathrm{~h}$, then placed the pouches with seeds on the germination pouch shelf in a temperature-controlled incubator at $25^{\circ} \mathrm{C}$ in dark. Five time points during seed germination including dry, 3 HAI (hours after imbibition), 6 HAI and 12 HAI and 24 HAI were investigated in the current study. For each time point, 20 healthy embryonic axes were selected and separated from the testing seed for RNA extractions [17].

\section{RNA extraction and quantitative RT-PCR validation}

Three independent plants consisting of three biological replicates were adopted for RNA extractions and quantitative RT-PCR validations. Total RNA was isolated from 
the samples by using the RNAprep pure plant kit (TIANGEN, Beijing, China) [17]. And the quality of extracted RNA was examined by electrophoresis and then quantified with a Nanodrop ND-1000 spectrophotometer (Nanodrop, Wilmington, DE, USA) [17]. We selected 17 representative GmPME genes and conducted the quantitative RT-PCR validations to ulteriorly explore the expression patterns of GmPME genes during soybean flower bud developments as well as seed germination. The specific quantitative RT-PCR primers were designed by Primer Premier 5 and listed in Table S17 (Additional file 19). The removement of genomic DNA and conversion of RNA to cDNA were conducted by using the HiScript II 1st Strand cDNA Synthesis Kit (Vazyme Biotech, Nanjing, China) [17]. A BioRad CFX96 real-time system was adopted to perform the quantitative RT-PCR assay with SYBR qPCR Master Mix (Vazyme Biotech, Nanjing, China) [17]. Triplicate quantitative assays were performed on each cDNA sample and analyzed by a $2^{-\triangle \triangle \mathrm{CT}}$ method by setting the housekeeping GmActin gene as the internal control [62].

\section{Statistical analyses}

Student's $t$-test was examined by Graphpad Prism 8 and the $P$-value cut-off of 0.05 was the criterion to determine whether the test was significantly different or not. Error bars in the column diagrams were standard deviations (SD) from the independent biological replicates.

\begin{abstract}
Abbreviations
aa: Amino acid; At: Arabidopsis thaliana; bp: Base pair; CDS: Coding sequence; Chr: Chromosome; CMS: Cytoplasmic male sterility; FPKM: Fragments per kilobase million; Gm: Glycine max; GMPME: Glycine max PME gene; Gs: Glycine soja; HAl: Hours after imbibition; Ka: Non-synonymous substitution; kb: Kilobase; Ks: Synonymous substitution; MW: Molecular weight; NJ: Neighbor-joining: ORF: Opening reading frame; Os: Oryza sativa; pl: Isoelectric point; PME: Pectin methylesterase; PMEI: PME inhibitor; SAM: Shoot apical meristem; Sb: Sorghum bicolor; SD: Standard deviation; SI: Solanum lycopersicum; UTRs: Untranslated regions; Vu: Vigna unguiculata; WGD: Whole genome duplication.
\end{abstract}

\section{Supplementary Information}

The online version contains supplementary material available at https://doi. org/10.1186/s12870-021-03355-1.

Additional file 1: Table S1. The 127 identified GMPME genes in this study. Additional file 2: Table S2. Coding sequences and protein sequences of the identified GMPME gene members.

Additional file 3: Table S3. The 66 AtPME genes in Arabidopsis.

Additional file 4: Table S4. Analyses the motifs in soybean PME proteins from the MEME website.

Additional file 5: Table S5. Gene density of each chromosome of the soybean genome.

Additional file 6: Table S6. The synonymous substitution calculations of soybean homologous genes.
Additional file 7: Table S7. The segmentally duplicated gene pairs throughout the soybean genome.

Additional file 8: Table S8. The tandemly duplicated gene pairs throughout the soybean genome.

Additional file 9: Table S9. Tandemly and segmentally duplicated GMPME gene pairs.

Additional file 10: Table S10. One-to-one orthologous relationships between the PME gene members in Glycine max and the other six species.

Additional file 11: Table S11. Non-redundant GmPME gene IDs associated with the syntenic relationships among soybean and the other six species.

Additional file 12: Table S12.Cis-element analysis of GMPME gene promoters.

Additional file 13: Table S13. Expression profiles of GMPME genes in multiple tissues on Phytozome.

Additional file 14: Table S14. Pairwise correlation coefficients between different expressed GMPME genes in various tissues.

Additional file 15: Table S15. Expression profiles of GMPME genes in soybean flower buds from cytoplasmic male sterile line NJCMS1A and its maintainer NJCMS1B.

Additional file 16: Figure S1. Phylogenetic expression profiles of GMPME genes in soybean flower buds from cytoplasmic male sterile line NJCMS1A and its maintainer NJCMS1B based on the published transcriptome data. The FPKM values were row-scaled with the zero-to-one method

Additional file 17: Table S16. Expression profiles of GmPME genes in soybean embryonic axes during seed germination.

Additional file 18: Figure S2. Phylogenetic expression profiles of the extracted GMPME genes in soybean embryonic axes during seed germination based on the reported transcriptome data. The FPKM values were row-scaled with the zero-to-one method. GMPME genes that lacked expression information in the transcriptome data were depicted with the gray color in the heatmap.

Additional file 19: Table S17. Sequences of the primers used in this study.

\section{Acknowledgments}

Not applicable.

\section{Authors' contributions}

SPY and LW conceived and designed the research. LW conducted the data analyses, experiments, and finished the original manuscript. LW, YQG, SMW and QQZ carried out the quantitative RT-PCR analyses. LW and SPY wrote and revised the manuscript. All the authors read and approved the final manuscript.

\section{Funding}

This study was funded by grants from the National Key R\&D Program of China (2016YFD0101500, 2016YFD0101504), the Fundamental Research Funds for the Central Universities (KYT201801), and the Program for Changjiang Scholars and Innovative Research Team in University (PCSIRT_17R55). These funding bodies took part in the design of the study and collection, analysis, and interpretation of data, and the writing of the manuscript, as well as in the open access payment.

\section{Availability of data and materials}

The reference genome of Glycine max used to identify the PME genes was released in JGI Phytozome 13 with the accession number of ACUP02000000 (https://phytozome-next.jgi.doe.gov/info/Gmax_Wm82_a2_v1). The genome and its annotation file of Arabidopsis thaliana were downloaded from JGI Phytozome 13 (https://phytozome-next.jgi.doe.gov/info/Athaliana_TAIR10). The genome and its annotation file of Glycine soja were downloaded from JGI Phytozome 13 (https://phytozome-next.jgi.doe.gov/info/Gsoja_v1_1). The genome and its annotation file of Vigna unguiculata were downloaded from JGI Phytozome 13 (https://phytozome-next.jgi.doe.gov/info/Nunguiculata_v1_1). The genome and its annotation file of Solanum lycopersicum were downloaded from 
JGI Phytozome 13 (https://phytozome-next.jgi.doe.gov/info/Slycopersicum_ ITAG3_2). The genome and its annotation file of Oryza sativa were downloaded from JGl Phytozome 13 (https://phytozome-next.jgi.doe.gov/info/Osativa_v7_0). The genome and its annotation file of Sorghum bicolor were downloaded from JGI Phytozome 13 (https://phytozome-next.jgi.doe.gov/info/Sbicolor_v3_1_1). The gene expression data in soybean various tissues were downloaded from Phytozome (https://phytozome.jgi.doe.gov/pz/portal.html). The RNA-Seq reads in flower buds of soybean cytoplasmic male sterile line NJCMS1A and its maintainer NJCMS1B were deposited in Sequence Read Archive database (http://www.ncbi. nlm.nih.gov/Traces/sra/) under the accession number SRP052011. The transcriptome datasets generated in soybean embryonic axis in five time points during germination were downloaded from GEO (https://www.ncbi.nlm.nih.gov/geo/ query/acc.cgi?acc $=$ GSE83481) under the accession number GSE83481. Soybean materials used in this study (NJCMS1A, NJCMS1B and Williams 82) were supplied by Soybean Research Institute, National Center for Soybean, Key Improvement Laboratory of Biology and Genetic Improvement of Soybean (General, Ministry of Agriculture), State Key Laboratory of Crop Genetics and Germplasm Enhancement, Jiangsu Collaborative Innovation Center for Modern Crop Production, College of Agriculture, Nanjing Agricultural University, Nanjing 210095, China. All data generated or analyzed during this study are included in this published article and its Additional files.

\section{Declarations}

Ethics approval and consent to participate

Not applicable.

\section{Consent for publication}

Not applicable.

\section{Competing interests}

The authors declare that they have no competing interests.

Received: 10 February 2021 Accepted: 17 November 2021

Published online: 06 December 2021

\section{References}

1. Cosgrove DJ. Plant cell wall extensibility: connecting plant cell growth with cell wall structure, mechanics, and the action of wall-modifying enzymes. J Exp Bot. 2016;67(2):463-76.

2. Xue C, Guan SC, Chen JQ, Wen CJ, Cai JF, Chen X. Genome wide identification and functional characterization of strawberry pectin methylesterases related to fruit softening. BMC Plant Biol. 2020;20(1):13.

3. Anderson CT. We be jammin': an update on pectin biosynthesis, trafficking and dynamics. J Exp Bot. 2016;67(2):495-502.

4. Daher FB, Braybrook SA. How to let go: pectin and plant cell adhesion. Front Plant Sci. 2015;6:523.

5. Huang YC, Wu HC, Wang YD, Liu CH, Lin CC, Luo DL, et al. PECTIN METHYLESTERASE34 contributes to heat tolerance through its role in promoting stomatal movement. Plant Physiol. 2017;174(2):748-63.

6. Wang DD, Yeats TH, Uluisik S, Rose JKC, Seymour GB. Fruit softening: revisiting the role of pectin. Trends Plant Sci. 2018;23(4):302-10.

7. Catoire L, Pierron M, Morvan C, du Penhoat CH, Goldberg R. Investigation of the action patterns of pectinmethylesterase isoforms through kinetic analyses and NMR spectroscopy: implications in cell wall expansion. J Biol Chem. 1998;273(50):33150-6.

8. Wang MJ, Yuan DJ, Gao WH, Li Y, Tan JF, Zhang XL. A comparative genome analysis of PME and PMEI families reveals the evolution of pectin metabolism in plant cell walls. PLoS One. 2013;8(8):e72082.

9. Bosch M, Cheung AY, Hepler PK. Pectin methylesterase, a regulator of pollen tube growth. Plant Physiol. 2005;138(3):1334-46.

10. Kim YJ, Jeong HY, Kang SY, Silva J, Kim EJ, Park SK, et al. Physiological importance of pectin modifying genes during rice pollen development. Int J Mol Sci. 2020;21(14):4840.

11. Müller K, Levesque-Tremblay $G$, Bartels $S$, Weitbrecht $K$, Wormit A, Usadel $B$, et al. Demethylesterification of cell wall pectins in Arabidopsis plays a role in seed germination. Plant Physiol. 2013;161(1):305-16.
12. Zúñiga-Sánchez E, Soriano D, Martínez-Barajas E, Orozco-Segovia A, Gamboa-deBuen A. BIIDXI, the At4g32460 DUF642 gene, is involved in pectin methyl esterase regulation during Arabidopsis thaliana seed germination and plant development. BMC Plant Biol. 2014;14(1):338.

13. Derbyshire P, McCann MC, Roberts K. Restricted cell elongation in Arabidopsis hypocotyls is associated with a reduced average pectin esterification level. BMC Plant Biol. 2007;7(1):31.

14. Pelletier S, Van Orden J, Wolf S, Vissenberg K, Delacourt J, Ndong YA, et al. A role for pectin de-methylesterification in a developmentally regulated growth acceleration in dark-grown Arabidopsis hypocotyls. New Phytol. 2010;188(3):726-39.

15. Lin XC, Yang R, Dou Y, Zhang W, Du HY, Zhu LQ, et al. Transcriptome analysis reveals delaying of the ripening and cell-wall degradation of kiwifruit by hydrogen sulfide. J Sci Food Agric. 2020;100(5):2280-7.

16. Wen B, Zhang F, Wu XZ, Li H. Characterization of the tomato (Solanum lycopersicum) pectin methylesterases: evolution, activity of isoforms and expression during fruit ripening. Front Plant Sci. 2020;11:238.

17. Wang L, Ding XL, Gao YQ, Yang SP. Genome-wide identification and characterization of GRAS genes in soybean (Glycine max). BMC Plant Biol. 2020;20(1):415.

18. Louvet R, Cavel E, Gutierrez L, Guénin S, Roger D, Gillet F, et al. Comprehensive expression profiling of the pectin methylesterase gene family during silique development in Arabidopsis thaliana. Planta. 2006;224(4):782-91.

19. Jeong HY, Nguyen HP, Lee CH. Genome-wide identification and expression analysis of rice pectin methylesterases: implication of functional roles of pectin modification in rice physiology. J Plant Physiol. 2015;183:23-9.

20. Zhang PP, Wang H, Qin XE, Chen K, Zhao JR, Zhao YX, et al. Genome-wide identification, phylogeny and expression analysis of the PME and PMEI gene families in maize. Sci Rep. 2019;9(1):19918.

21. Li WJ, Shang HH, Ge Q, Zou CS, Cai J, Wang DJ, et al. Genome-wide identification, phylogeny, and expression analysis of pectin methylesterases reveal their major role in cotton fiber development. BMC Genomics. 2016:17(1):1000.

22. Guénin S, Hardouin J, Paynel F, Müller K, Mongelard G, Driouich A, et al. AtPME3, a ubiquitous cell wall pectin methylesterase of Arabidopsis thaliana, alters the metabolism of cruciferin seed storage proteins during post-germinative growth of seedlings. J Exp Bot. 2017;68(5):1083-95.

23. Del Corpo D, Fullone MR, Miele R, Lafond M, Pontiggia D, Grisel S, et al. AtPME17 is a functional Arabidopsis thaliana pectin methylesterase regulated by its PRO region that triggers PME activity in the resistance to Botrytis cinerea. Mol Plant Pathol. 2020;21(12):1620-33.

24. Yue $X Y$, Lin $S, Y u Y J$, Huang L, Cao JS. The putative pectin methylesterase gene, BCMF23a, is required for microspore development and pollen tube growth in Brassica campestris. Plant Cell Rep. 2018;37(7):1003-9.

25. Xiong XP, Zhou D, Xu LA, Liu TT, Yue XY, Liu WM, et al. BCPME37C is involved in pollen intine formation in Brassica campestris. Biochem Biophys Res Commun. 2019;517(1):63-8.

26. Wang $L$, Cheng YB, Ma QB, Mu YH, Huang ZF, Xia QJ, et al. QTL fine-mapping of soybean (Glycine max L.) leaf type associated traits in two RILs populations. BMC Genomics. 2019;20(1):260.

27. Wang JJ, Ling L, Cai H, Guo CH. Gene-wide identification and expression analysis of the PMEl family genes in soybean (Glycine max). 3 Biotech. 2020;10(8):335.

28. Kim KS, Diers BW, Hyten DL, Rouf Mian MA, Shannon JG, Nelson RL. Identification of positive yield QTL alleles from exotic soybean germplasm in two backcross populations. Theor Appl Genet. 2012;125(6):1353-69.

29. Ding XL, Li JJ, Zhang H, He TT, Han SH, Li YW, et al. Identification of miRNAs and their targets by high-throughput sequencing and degradome analysis in cytoplasmic male-sterile line NJCMS1A and its maintainer NJCMS1B of soybean. BMC Genomics. 2016;17(1):24

30. Li YW, Ding XL, Wang X, He TT, Zhang H, Yang LS, et al. Genome-wide comparative analysis of DNA methylation between soybean cytoplasmic male-sterile line NJCMS5A and its maintainer NJCMS5B. BMC Genomics. 2017;18(1):596.

31. Yuan FJ, Yu XM, Dong DK, Yang QH, Fu XJ, Zhu SL, et al. Whole genome-wide transcript profiling to identify differentially expressed genes associated with seed field emergence in two soybean low phytate mutants. BMC Plant Biol. 2017;17(1):16 
32. Moore RC, Purugganan MD. The early stages of duplicate gene evolution. Proc Natl Acad Sci U S A. 2003;100(26):15682-7.

33. Schmutz J, Cannon SB, Schlueter J, Ma JX, Mitros T, Nelson W, et al. Genome sequence of the palaeopolyploid soybean. Nature. 2010;463(7278):178-83.

34. Cannon SB, Mitra A, Baumgarten A, Young ND, May G. The roles of segmental and tandem gene duplication in the evolution of large gene families in Arabidopsis thaliana. BMC Plant Biol. 2004:4(1):10.

35. Zhu Y, Wu NN, Song WL, Yin GJ, Qin YJ, Yan YM, et al. Soybean (Glycine max) expansin gene superfamily origins: segmental and tandem duplication events followed by divergent selection among subfamilies. BMC Plant Biol. 2014;14(1):93.

36. Yu J, Wang J, Lin W, Li SG, Li H, Zhou J, et al. The genomes of Oryza sativa: a history of duplications. PLoS Biol. 2005;3(2):e38.

37. Holub EB. The arms race is ancient history in Arabidopsis, the wildflower. Nat Rev Genet. 2001;2(7):516-27.

38. Xie T, Chen CJ, Li CH, Liu JR, Liu CY, He YH. Genome-wide investigation of WRKY gene family in pineapple: evolution and expression profiles during development and stress. BMC Genomics. 2018;19(1):490.

39. Melchinger AE. The international conference on "heterosis in plants". Theor Appl Genet. 2009:120(2):201.

40. Li JJ, Han SH, Ding XL, He TT, Dai JY, Yang SP, et al. Comparative transcriptome analysis between the cytoplasmic male sterile line NJCMS1A and its maintainer NJCMS1B in soybean (Glycine max (L.) Merr.). PLoS One. 2015;10(5):e0126771.

41. Sangi S, Santos MLC, Alexandrino CR, Da Cunha M, Coelho FS, Ribeiro GP, et al. Cell wall dynamics and gene expression on soybean embryonic axes during germination. Planta. 2019;250(4):1325-37.

42. Bellieny-Rabelo D, de Oliveira EAG, Ribeiro ES, Costa EP, Oliveira AEA, Venancio TM. Transcriptome analysis uncovers key regulatory and metabolic aspects of soybean embryonic axes during germination. Sci Rep. 2016:6(1):36009.

43. Liu QX, Talbot M, Llewellyn DJ. Pectin methylesterase and pectin remodelling differ in the fibre walls of two Gossypium species with very different fibre properties. PLoS One. 2013;8(6):e65131.

44. Micheli F. Pectin methylesterases: cell wall enzymes with important roles in plant physiology. Trends Plant Sci. 2001;6(9):414-9.

45. Pinzón-Latorre D, Deyholos MK. Characterization and transcript profiling of the pectin methylesterase (PME) and pectin methylesterase inhibitor (PMEI) gene families in flax (Linum usitatissimum). BMC Genomics. 2013;14(1):742.

46. Guo RR, Xu XZ, Carole B, Li XQ, Gao M, Zheng Y, et al. Genome-wide identification, evolutionary and expression analysis of the aspartic protease gene superfamily in grape. BMC Genomics. 2013;14(1):554.

47. Li FG, Fan GY, Lu CR, Xiao GH, Zou CS, Kohel RJ, et al. Genome sequence of cultivated Upland cotton (Gossypium hirsutum TM-1) provides insights into genome evolution. Nat Biotechnol. 2015;33(5):524-30.

48. William Roy S, Gilbert W. The evolution of spliceosomal introns: patterns, puzzles and progress. Nat Rev Genet. 2006;7(3):211-21.

49. Roy SW, Penny D. A very high fraction of unique intron positions in the intron-rich diatom Thalassiosira pseudonana indicates widespread intron gain. Mol Biol Evol. 2007;24(7):1447-57.

50. Iwamoto M, Maekawa M, Saito A, Higo H, Higo K. Evolutionary relationship of plant catalase genes inferred from exon-intron structures: isozyme divergence after the separation of monocots and dicots. Theor Appl Genet. 1998;97(1):9-19

51. Sun M, Zhang M, Singh J, Song B, Tang Z, Liu Y, et al. Contrasting genetic variation and positive selection followed the divergence of NBS-encoding genes in Asian and European pears. BMC Genomics. 2020:21(1):809.

52. Suzuki H, Rodriguez-Uribe L, Xu JN, Zhang JF. Transcriptome analysis of cytoplasmic male sterility and restoration in CMS-D8 cotton. Plant Cell Rep. 2013;32(10):1531-42.

53. Luján-Soto E, Dinkova TD. Time to wake up: epigenetic and small-RNAmediated regulation during seed germination. Plants. 2021;10(2):236.

54. Ren C, Kermode AR. An increase in pectin methyl esterase activity accompanies dormancy breakage and germination of yellow cedar seeds. Plant Physiol. 2000;124(1):231-42.

55. Nakabayashi K, Okamoto M, Koshiba T, Kamiya Y, Nambara E. Genomewide profiling of stored mRNA in Arabidopsis thaliana seed germination: epigenetic and genetic regulation of transcription in seed. Plant J. 2005;41(5):697-709
56. Chen CJ, Chen H, Zhang Y, Thomas HR, Frank MH, He YH, et al. TBtools: an integrative toolkit developed for interactive analyses of big biological data. Mol Plant. 2020:13(8):1194-202.

57. Kumar S, Stecher G, Tamura K. MEGA7: molecular evolutionary genetics analysis version 7.0 for bigger datasets. Mol Biol Evol. 2016;33(7):1870-4.

58. Edgar RC. MUSCLE: multiple sequence alignment with high accuracy and high throughput. Nucleic Acids Res. 2004;32(5):1792-7.

59. Krzywinski M, Schein J, Birol I, Connors J, Gascoyne R, Horsman D, et al. Circos: an information aesthetic for comparative genomics. Genome Res. 2009;19(9):1639-45.

60. Lescot M, Déhais P, Thijs G, Marchal K, Moreau Y, Van de Peer Y, et al. PlantCARE, a database of plant cis-acting regulatory elements and a portal to tools for in silico analysis of promoter sequences. Nucleic Acids Res. 2002;30(1):325-7.

61. Bai YN, Gai JY. Inheritance of male fertility restoration of the cytoplasmicnuclear male-sterile line NJCMS1A of soybean [Glycine max (L) Merr.]. Euphytica. 2005;145(1):25-32.

62. Livak KJ, Schmittgen TD. Analysis of relative gene expression data using realtime quantitative PCR and the $2^{-\Delta \Delta C T}$ method. Methods. 2001;25(4):402-8.

\section{Publisher's Note}

Springer Nature remains neutral with regard to jurisdictional claims in published maps and institutional affiliations.

Ready to submit your research? Choose BMC and benefit from

- fast, convenient online submission

- thorough peer review by experienced researchers in your field

- rapid publication on acceptance

- support for research data, including large and complex data types

- gold Open Access which fosters wider collaboration and increased citations

- maximum visibility for your research: over $100 \mathrm{M}$ website views per year

At BMC, research is always in progress.

Learn more biomedcentral.com/submissions 